\title{
The Fourth Workshop on Lineshape Code Comparison: Line Merging
}

\author{
Spiros Alexiou ${ }^{1, *,+}$, Evgeny Stambulchik ${ }^{2}$ (D), Thomas Gomez ${ }^{3}$ and Mohammed Koubiti ${ }^{4}$ \\ TETY, University of Crete, 71409 Heraklion, TK 2208, Greece \\ 2 Faculty of Physics, Weizmann Institute of Science, Rehovot 7610001, Israel; \\ evgeny.stambulchik@weizmann.ac.il \\ 3 Sandia National Laboratories, Albuquerque, NM 87123, USA; thogome@sandia.gov \\ 4 Aix Marseille University, CNRS, PIIM, 13007 Marseille, France; mohammed.koubiti@univ-amu.fr \\ * Correspondence: moka1@otenet.gr; Tel.: +30-698-461-3962 \\ † Current address: Hellenic Army Academy, Varis, Koropiou Avenue Vari P.O. 16673, Greece.
}

Received: 28 February 2018; Accepted: 29 March 2018; Published: 31 March 2018

\begin{abstract}
For a given set of plasma parameters, along a single series (Lyman, Balmer, etc.) the lines with higher principal quantum number $(n)$ lines get progressively wider, closer to each other, and start merging for a certain critical $n$. In the present work, four different codes (with further options) are used to calculate the entire Balmer series for moderate and high electron densities. Particular attention is paid to the relevant physics, such as the cutoff criteria, strong and penetrating electron collisions.
\end{abstract}

Keywords: plasma spectroscopy; Line broadening; series limit; Balmer lines; Inglis-Teller limit; line merging

\section{Introduction: Series and Line Merging}

For a given set of plasma parameters, the states involved for the higher series members become progressively more strongly perturbed. This leads to wider and wider lines as the principal quantum number $n$ of the upper level increases. Ultimately these lines merge into the continuum and are no longer observable as discrete transitions. This effect is used for plasma diagnostics [1]. There are a number of factors resulting in such states [2] losing their atomic character with the sharply defined energy, e.g.,:

1. The line width (HWHM) compared to the distance of the line center to the next line or the continuum. The first is the usual ${ }^{1}$ Inglis-Teller criterion [1] used to determine the maximum $n$ in the series. Figure 1 illustrates the second criterion (HWHM compared to the distance from the line center to the continuum) by showing a case where the HWHM is larger than the distance of the line center to the continuum. In principle, the same applies to the shift, i.e., the unperturbed position's distance from the next level or the continuum is less than the shift; however for the Hydrogen Balmer series considered in this workshop, this was not an issue since the shift (not computed for the majority of participating codes) is much smaller than the width.

2. Wavefunction extent considerations: Another reason why a state stops being an atomic state with a sharply defined energy is if its wavefunction extent is comparable to the interparticle distance. An electron wavefunction with such a spatial extent is shared by two or more instead of

1 Except that in contrast to the original Inglis-Teller which used an estimate for the line width, the codes using it compute the line width by a full electron-ion broadening calculation. 
one center [3]. Ultimately when all centers are taken into account, the electron wavefunction is deformed so that the electron belongs to all centers, i.e., the plasma as a whole. This means that such an electron can be found a macroscopic distance away from the first center with no change in energy, i.e., it is essentially a free, not a bound, electron.

\section{$\mathrm{H}-12$ at the lowest density in PST}

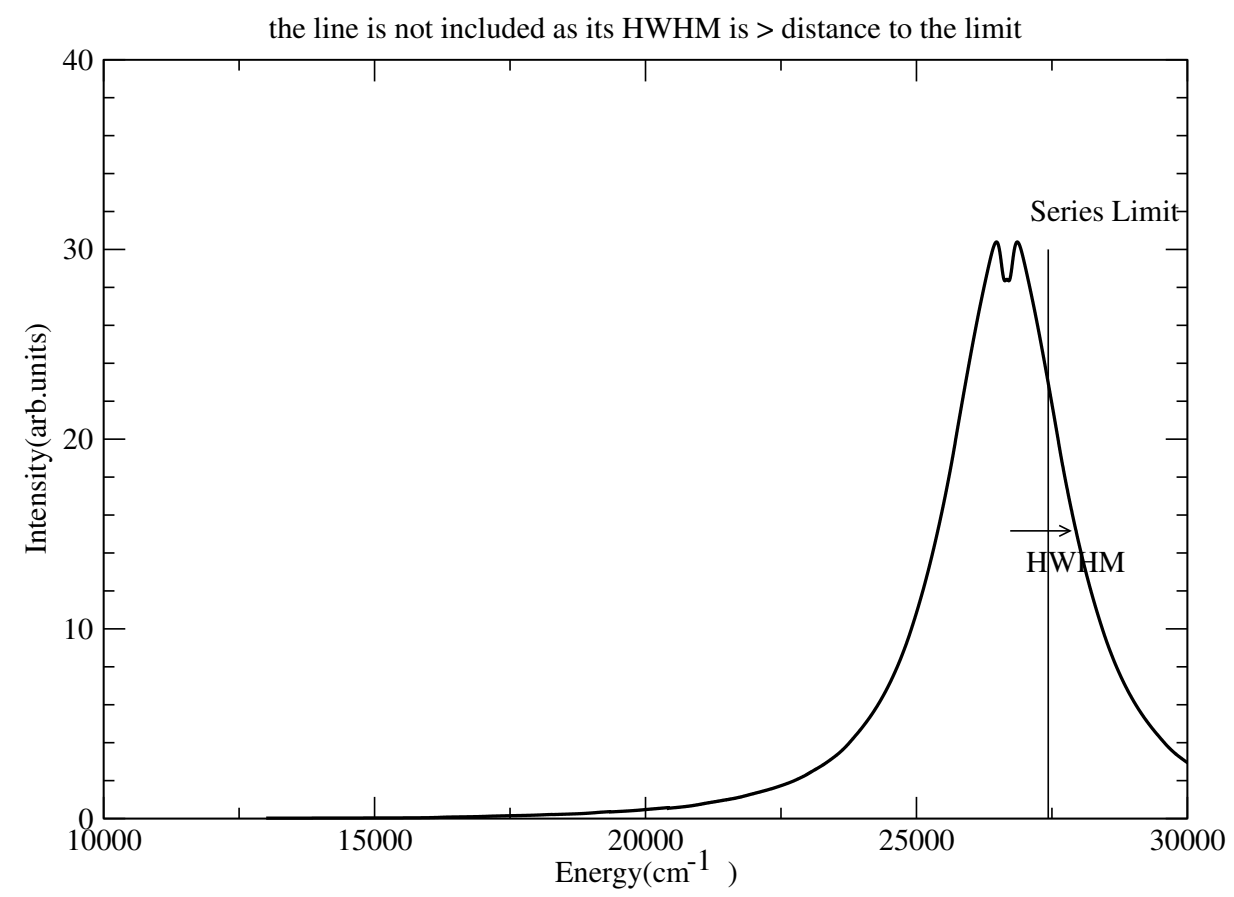

Figure 1. $H_{12}$ computed with account of penetrating collisions (PST) at the lowest density considered showing a HWHM that is larger than the distance to the continuum.

In the workshop calculations were requested at a fixed temperature $\mathrm{T}=1 \mathrm{eV}$ and three different electron densities: $N=3 \times 10^{17}, N=10^{18}$ and $N=3 \times 10^{18} \mathrm{e} / \mathrm{cm}^{3}$.

\section{Relevant Physics}

Table 1 summarizes some key physics.

Table 1. Line broadening issues and their importance for line mergings.

\begin{tabular}{ccc}
\hline Issue & Importance $\mathbf{( Y / N )}$ & Comment \\
\hline Ion Dynamics & $\mathrm{N}$ & For the entire series \\
Strong Collisions & $\mathrm{Y}$ & For the high $n$-lines \\
Penetrating Collisions & $\mathrm{Y}$ & For the high $n$-lines \\
Quantum effects & $\mathrm{N}$ & Could be for lower temperatures \\
\hline
\end{tabular}

\subsection{Ion Dynamics}

Ion dynamics is expected to be largely unimportant, especially for the higher series members. This is due to two reasons: First, higher series members involve wavefunctions that have larger spatial extents and hence high polarizability. This means that because of the stronger interaction with the plasma, the autocorrelation function (the Fourier transform of the line shape) decays on a short time scale, short enough that ions have no time to change their field appreciably. Second, even for the lines that do have a central component and are thus much more succeptible to ion dynamics effects, the relative intensity of that component decreases with increasing principal quantum number. Hence 
the most significant effects are expected for the $H_{\alpha}$ line at the lowest density. Even for that case however, calculations with full account for ion dynamics show that even for $H_{\alpha}$ at the lowest density considered, ion dynamics is not important, as illustrated in Figure 2; hence it can be safely ignored for the entire series at the plasma parameters considered in this work.

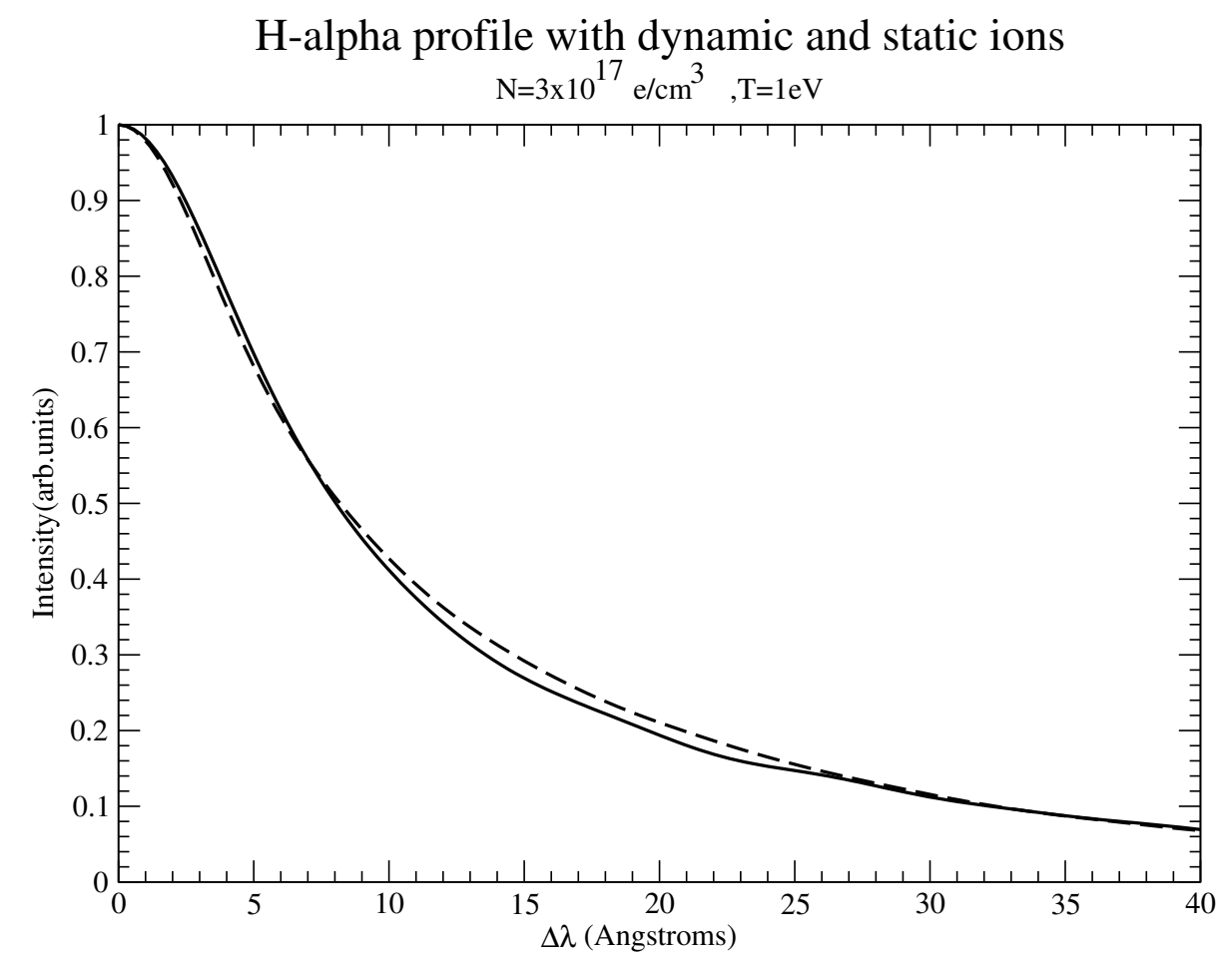

Figure 2. $H_{\alpha}$ profile with dynamic (solid) and static (dashed) ions (protons) for a temperature $\mathrm{T}=1 \mathrm{eV}$ and electron density $N=3 \times 10^{17} \mathrm{e} / \mathrm{cm}^{3}$. The dynamic profile is computed by a joint electron-ion simulation, while the static ion profile is computed by convolution of the dynamic (simulation) electron profile with the quastistatic ion profile.

\subsection{Strong Collisions and Penetration}

The term "strong collisions" has been used in the literature to denote either "collisions that may not be treated by perturbation theory" or "collisions that may not be treated within the model used", e.g., collisions for which the semiclassical approximation is not valid or collisions for which the normally employed long-range approximation is not valid. Note that the two definitions may be in conflict. Consider, for example, penetrating collisions i.e., close collisions which penetrate the wavefunction extent. Such collisions may be non-perturbative within the (inappropriate in this case) long-range approximation and perturbative if the long-range approximation is not used. Specifically, the weak, large velocity and large impact parameter contribution is proportional in the impact approximation to the logarithm of maximum impact parameter $\rho_{\max }$ to the relevant wavefunction extent, estimated as $\rho_{\text {min }}=n^{2} a_{0}$, with $n$ the upper level principal quantum number and $a_{0}$ the Bohr radius. The maximum impact parameter is taken to be $\rho_{\max }=0.68 \lambda_{D}$ with $\lambda_{D}=\sqrt{\frac{\epsilon_{0} k T}{e^{2} N}}$ the Debye length. The logarithm should be compared to the strong collision term, which is of the order of 1 . Hence, unless $\ln \frac{\rho_{\max }}{\rho_{\min }} \gg 1$, strong collisions are important.

In Table $2, \ln \frac{\rho_{\max }}{\rho_{\min }}$ is listed for all lines that are included in the calculation based on the criteria outlined above. It is seen that for the higher series members in all cases, the maximum impact parameter is comparable to the relevant wavefunction extent. Hence, strong collisions and penetration are potentially important, at least for the higher series members [4]. 
Table 2. Relative importance of penetration for all lines in the series and plasma parameters considered in the workshop examples. $\ln \left(\frac{\rho_{\max }}{\rho_{\min }}\right)$ is displayed as a function of the electron density $N$ for all series lines considered in the bound-bound spectrum calculation. The density $N$ is expressed in units of $10^{17} \mathrm{e} / \mathrm{cm}^{3}$. Bold is used for cases where the strong collision term is comparable to or dominates the weak collision contribution.

\begin{tabular}{ccccccccccc}
\hline $\boldsymbol{N}$ & $\boldsymbol{H}_{\boldsymbol{\alpha}}$ & $\boldsymbol{H}_{\boldsymbol{\beta}}$ & $\boldsymbol{H}_{\gamma}$ & $\boldsymbol{H}_{\boldsymbol{\delta}}$ & $\boldsymbol{H}_{\boldsymbol{\epsilon}}$ & $\boldsymbol{H}_{\mathbf{8}}$ & $\boldsymbol{H}_{\mathbf{9}}$ & $\boldsymbol{H}_{\mathbf{1 0}}$ & $\boldsymbol{H}_{\mathbf{1 1}}$ & $\boldsymbol{H}_{\mathbf{1 2}}$ \\
\hline 3 & 2.96 & 2.39 & 1.94 & 1.58 & 1.27 & $\mathbf{1}$ & $\mathbf{0 . 7 7}$ & $\mathbf{0 . 5 6}$ & $\mathbf{0 . 3 6 5}$ & $\mathbf{0 . 1 9}$ \\
10 & 2.36 & 1.79 & 1.34 & $\mathbf{0 . 9 7}$ & $\mathbf{0 . 6 7}$ & $\mathbf{0 . 4}$ & $\mathbf{0 . 1 6 5}$ & & & \\
30 & 1.81 & 1.24 & $\mathbf{0 . 7 9}$ & $\mathbf{0 . 4 3}$ & $\mathbf{0 . 1 2}$ & & & & & \\
\hline
\end{tabular}

\subsection{Quantum Effects}

Quantum effects, i.e., problems with a classical path treatment of the plasma electrons are usually associated with a large electron de Broglie wavelength compared to the relevant impact parameters and a significant energy transfer in collisional excitation-deexcitation processes (backreaction). In the present work backreaction is thought to be unimportant because generally quenching collisions were not explicitly considered, i.e., the result of collisions of plasma electron with an emitter electron in the upper and lower level respectively is a transition of the emitter electron to another state with the same principal quantum number. Hence the importance of quantum effects with principal quantum number depends on whether the impact parameters contributing most to broadening are larger or smaller for the higher $n$ series members and due to their larger phase space, large impact parameters typically dominate for hydrogen.

\section{The Codes}

Four codes participated in the comparison: QC-FFM and PPP are codes based on the Frequency Fluctuation Model (FFM) [5,6]. Briefly, the quasi-contiguous (QC) model [7] uses a rectangular approximation for the static lineshape. QC-FFM [8] improves by using the FFM to add ion dynamics on top of the quasi-contiguous model. QC-FFM does not recognize or treat strong collisions; instead it uses FFM to account for the dynamics of both electrons and ions. Among the participating codes, QC-FFM and PPP include ion dynamics; the other codes assume purely static ions.

CONV uses a standard calculation of impact electrons and quasistatic ions, with (PST) or without (ST) account for penetrating collisions. In addition, for each ST and PST $[9,10]$ calculations, runs were contributed with or without account of strong collisions. In CONVST, strong are collisions with impact parameters that are either smaller than $n^{2} a_{0}$ or violate unitarity in a perturbative treatment for a given velocity. In CONVPST, strong collisions are slow enough collisions that violate unitarity in a perturbative treatment accounting for penetration [9]. In either case, if strong collisions are accounted for, a strong collision term corresponding to the assumption that for impact parameters $\rho$ and velocities $v$ in the strong collision phase space, the angular averaged matrix $Q=\left\{1-S_{a}(\rho, v) S_{b}^{*}(\rho, v)\right\}=1$, i.e., the matrix in question is purely diagonal and unity for all $\rho, v$ in the strong collision phase space (other choices, e.g., 0.5 or 0.25 are, of course, possible). $S_{a}$ and $S_{b}$ denote S-matrices for the upper and lower levels respectively. Thus, apart from differences in the computation of electron broadening, differences between the QC-FFM and PPP on the one hand and CONV on the other is expected to be due to the treatment of ion dynamics, as a corresponding option in CONV was not used. Ion dynamics was discussed above and shown to be unimportant for all lines and densities in question, and expected to be unimportant for line-merging situations in general, as already discussed. Although CONV is a single code, we refer to its ST (CONVST) and PST (CONVPST) results as if they were different codes in the remainder of this work. CONV has a number of options and relevant ones are discussed later on. Finally DWE $[11,12]$ is a spectral Line broadening model that treats plasma electrons quantum mechanically using a second-order distorted wave (DW) treatment. Exchange between the plasma electrons and the bound electron(s) is included. The theory is based on the relaxation theory of Fano 
and is expanded from the work of Woltz and Hooper [13]. The interaction potential is a full-Coulomb potential, which includes penetration and monopolar, dipolar and quadrupolar interactions. The ions are still treated classically: For charged radiators (not considered here) they are treated in the usual dipole approximation with no penetration and only for neutral hydrogen are the ions treated with full Coulomb interaction. The electron calculation includes penetrating collisions and therefore includes monopolar interactions as well as reconnection term. In this respect DWE is close to CONVPST, in that penetration is included, but differs from all other treatments in its quantum-mechanical treatment of perturbing (plasma) electrons, although PPP also uses an electron broadening (collision) operator that is also based on a distorted wave calculation [14]. The main difference from DWE is that the Griem, Blaha and Kepple [14] used in PPP is tailored for highly-charged Z and does not include exchange, while DWE does. As shown in [11,12], exchange makes a large difference for low Z-elements and this difference is exacerbated for high densities; in contrast plane or distorted wave calculation differences are much smaller.

In terms of the differences in the results of quantal and semiclassical approaches, the most important factor that is taken into account in quantal calculations and normally neglected in semiclassical calculations is penetration into the emitter wavefunctions [15]. This effect softens the interaction and makes perturbative treatments valid for a larger part of the phase space, resulting in a much reduced strong collision term and relatively small difference (practically zero for the present work) in the CONVPST calculations with and without the strong collision term. As a result for hydrogen and $\mathrm{H}$-like lines, this effect can be accounted for analytically and this has been done for the dipole part of the interaction $[2,9,10]$. It is, therefore, probably not a coincidence that agreement between CONVPST and DWE is very good for all lines in the series. This is illustrated in Figures 3 and 4 for the intermediate density considered. For comparison PPP and QC-FFM are also plotted. Interestingly enough, QC-FFM, which does not account for strong or penetrating collisions agrees best among the remaining codes with CONVPST and DWE.

\section{DWE, CONVPST series at the intermediate density}

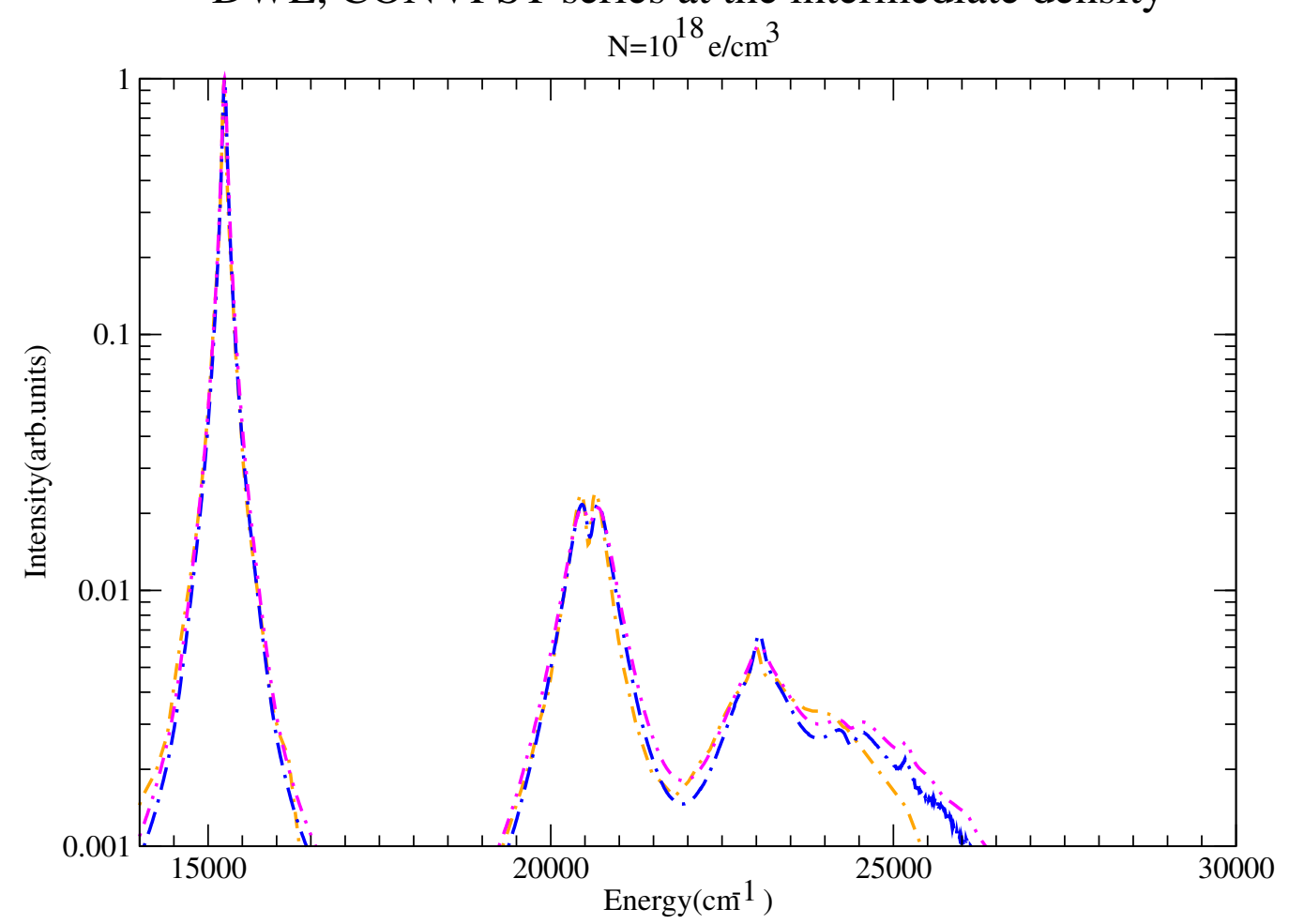

Figure 3. The entire series for the intermediate density considered for DWE (dash-dotted orange) and CONVPST without (long dash-dotted blue) and with strong collisions (dash-double dotted magenta). 
DWE, CONVPST at the intermediate density: H-gamma and beyond

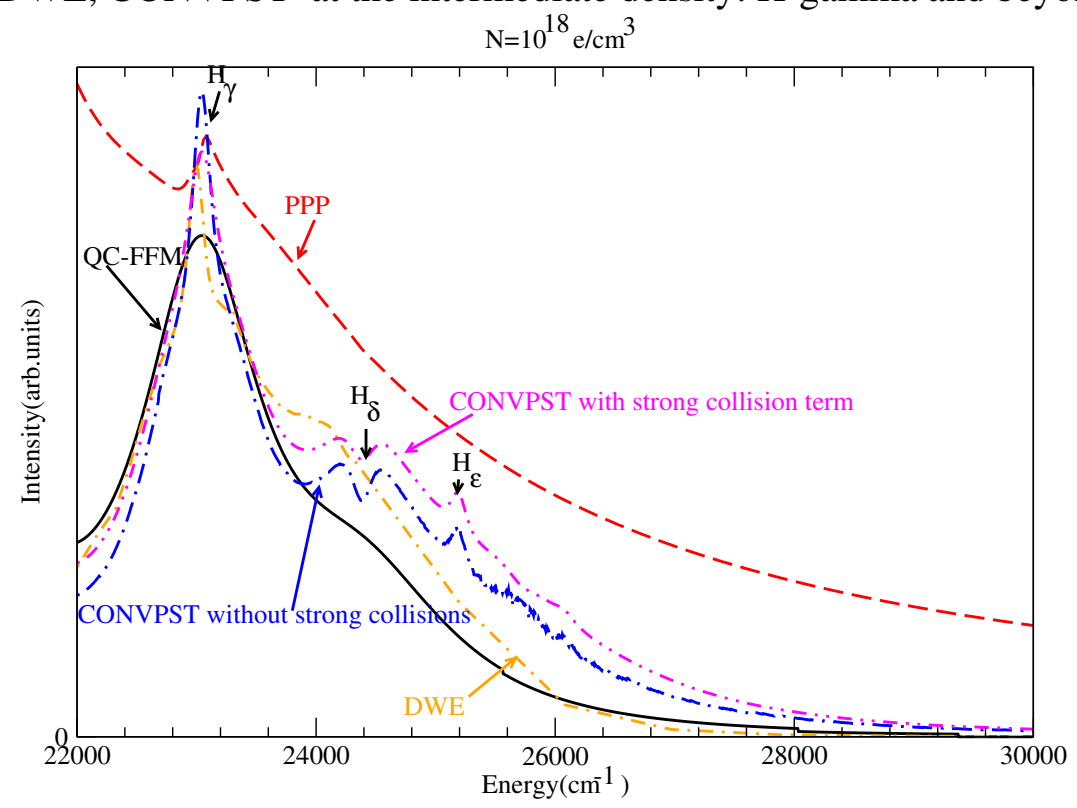

Figure 4. The series from $H_{\gamma}$ to the continuum for the intermediate density considered for DWE (dash-dotted orange) and CONVPST without (long dash-dotted blue) and with strong collisions (dash-double dotted magenta). For comparison PPP (red dashed) and QC-FFM (solid black) are also shown.

\subsection{Treatment of Strong Collisions in the Codes}

As already mentioned, CONV and PPP (via the electron collision operator [14]) include a strong collision term. CONV adds a strong collision term of $\pi N \rho_{\text {max }}^{2}\langle v\rangle$ times a strong collision parameter $\mathrm{Q}$ which is between 0 and 2, corresponding to the approximation that for strong collisions $Q=1-S_{a}(\rho, v) S_{b}^{*}(\rho, v)=0$ or 2 respectively for all impact parameters $\rho$ and velocities $v$, respectively. In normal runs, this parameter is taken to be the average, i.e., 1 . In runs without strong collisions, this is taken to be 0 . Note that for introducing appropriate unitarity cutoffs, the unitarity cutoff $\rho_{\min }(v)$ is determined by setting $I-S_{a}(\rho, v) S_{b}^{*}(\rho, v)=1$, whether the resulting strong collision phase space contributes or not (i.e., whether $Q=0$ or not). DWE does not make any distinction between strong and weak collisions. A full-Coulomb interaction with quantum-mechanical electrons is used in a distorted waves (DW) approximation. QC-FFM also has no strong collision term.

\subsection{The Last Bound Level}

QC-FFM continues going up in $n$ until the Stark width (HWHM) exceeds the distance to the next level (Inglis-Teller). Then the last bound-bound line's width is used to convolve with the free-bound spectrum which begins at the position of the $(n+1)$ th line of the series. CONVST/PST has a number of options to deal with this. For most runs (i.e., unless specified otherwise), CONVST/PST uses the distance to the continuum instead of the distance to the next level, but may (and does) stop at a smaller $n^{*}$ if:

- $\quad$ a. The wavefunction extent $n^{2} a_{0}$ is larger than the interatomic distance.

- b. The minimum impact parameter is larger than the maximum impact parameter (e.g., all collisions are strong) for CONVST.

DWE and PPP made no attempt to estimate if a state is bound or not. Instead, a large enough set of states was used. For PPP the maximum upper level principal quantum number $n^{*}$ was 15 for all runs. For DWE for the present comparison $n^{*}=7$ for all cases, except the highest density case, where it was 6 . This was imposed by Computational Storage considerations. The problem (i.e., the $U$-matrix) is 
then solved in the DW approximation in the basis set consisting of all states of $n=1,2,3,4,5,6$ and 7 for the lowest and intermediate density. The code differences in the choice of the last bound level are manifested in differences in the high energy part of the spectrum. As illustrated in Figure 4, the code predictions and underlying "last level" models (e.g., Inglis-Teller) can in principle by experimentally tested, e.g., based on plasma parameters determined from the lower-lying lines, it may be possible to distinguish experimentally the last level for these parameters.

\subsection{Line Wings}

CONVST/PST is a pure impact code; As a result incomplete collisions and their effect on the line wings are not correctly accounted for; however, an option to use $\rho_{\text {max }}$ scaled by $\frac{\omega_{p l}}{|\Delta \omega|}$ for $|\Delta \omega|>\omega_{p l}$ to account for incomplete collisions for short times, i.e., the Lewis cutoff [16], is available, although it results in a slightly abrupt change at $|\Delta \omega|=\omega_{p l}$, the plasma frequency. The idea is that for short enough times the electric field correlation function that enters the impact approximation has a time dependence $\left\langle\mathbf{E}(0 \cdot \mathbf{E}(t)\rangle \approx \frac{e^{-v t / \lambda_{D}}}{t}\right.$ and as a result the Coulomb logarithm $\ln \frac{\rho_{\max }}{\rho_{\min }}$ is replaced by $\ln \frac{v}{|\Delta \omega| \rho_{\min }}$, i.e., the maximum impact parameter $\rho_{\text {max }}$ of the order of the Debye length $\lambda_{D}=\langle v\rangle / \omega_{p l}$ is replaced by $\frac{\langle v\rangle}{\omega_{p l}} \frac{\omega_{p l}}{|\Delta \omega|}$, i.e., for $|\Delta \omega|>\omega_{p l}$, the maximum impact parameter is effectively multiplied by $f=\frac{\omega_{p l}}{|\Delta \omega|}$. Since the collision operator $\phi$ and strong collision term are both integrals with the maximum impact parameter as the upper limit, i.e., of $f$, the code computes the ratio of the collision operator $\phi(f) / \phi(1)$ as well as the corresponding ratio of strong collision terms a function of $f$ which ranges from 0 to 1 and uses these interpolation tables to obtain the necessary quantities at any given $\Delta \omega$. The interpolation is illustrated for both ST and PST in Figure 5 which plots the scaling factor $\phi(f) / \phi(1)$ for the collision operator $\phi$ vs. $f=\frac{\omega_{p l}}{|\Delta \omega|}$, which is the shrinking factor for the maximum impact parameter as discussed above. For PST, such a table is created for each "channel", even though only one is shown in Figure 5. A similar interpolation table is created and used for the strong collision term, which is again a function of the maximum impact parameter $\rho_{\max }$.

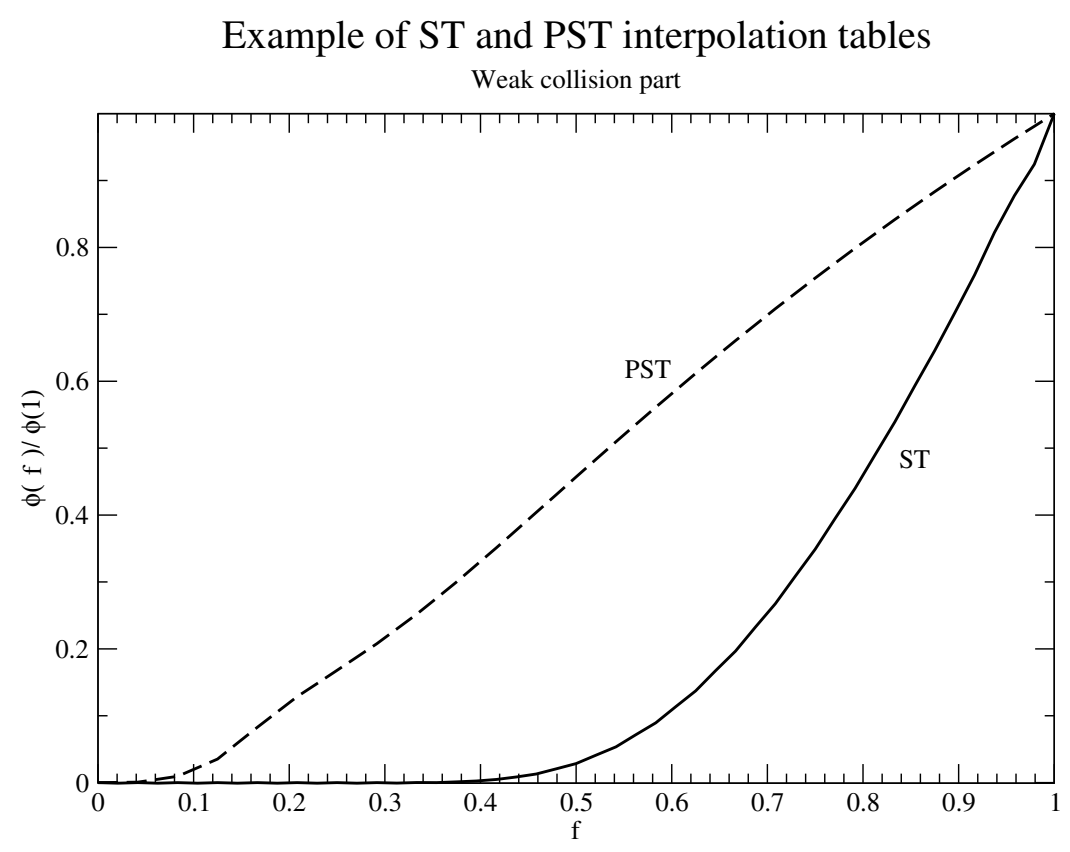

Figure 5. Illustration of Lewis cutoff interpolation table for both ST (solid) and a single PST "channel" (dashed).

The results of using the Lewis cutoff are illustrated in Figure 6 for the $H_{\alpha}$ line at the intermediate density. Unless specified otherwise, this option was not used for most runs. 
Lewis cutoff for the intermediate density: $\mathrm{H}$-alpha

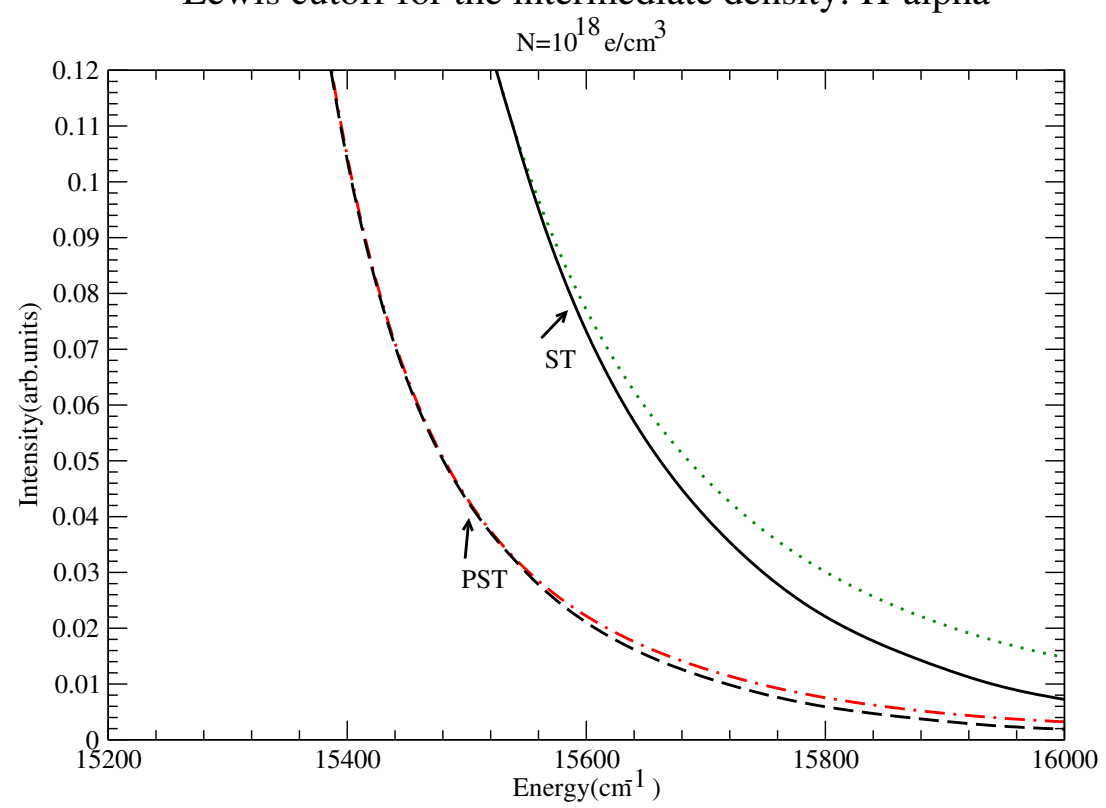

Figure 6. $H_{\alpha}$ for CONVST and CONVPST with and without the Lewis cutoff for the intermediate density. Shown are CONVST with Lewis cutoff (black solid), CONVST without Lewis cutoff (green dotted), CONVPST with Lewis cutoff (black dashed) and CONVPST without Lewis cutoff (red dash-dotted).

QC-FFM recovers the quasistatic wings nicely, by construction (just as FFM does). PPP uses the Griem-Blaha-Kepple broadening operator and with no frequency dependence, i.e., is also a pure impact code. There is the possibility to include frequency dependence in the broadening operator in post-processing [17], but this was not used in the present runs and is consistent with the overestimation of the widths and wing intensities. DWE is based on Fano's relaxation formalism, which takes into account the frequency dependence of the broadening operator and in that sense, it is closer to the unified theory rather than the impact theory.

Tables 3 and 4 address line wing issues for CONVST/PST. The line widths (HWHM) for all but the lowest series members are seen to be larger than the plasma frequency, hence invalidating the impact theory used ( the unified theory should be used instead). Hence, even the bulk of the line, not just the line wings are affected. Despite this fact, the Lewis cutoff option was not used in CONV for the bulk of the runs, in order to illustrate the importance of the wing lineshape.

Table 3. Widths vs. plasma frequency $\omega_{p l}$ and distance $D$ to the continuum for CONVST with strong collisions as a function of the upper level principal quantum number $n$. All energies are in $\mathrm{cm}^{-1}$ and densities $N$ in $10^{17} \mathrm{e} / \mathrm{cm}^{3}$. The 'Bound' column indicates whether the state is considered bound (Y) or $\operatorname{not}(\mathrm{N})$.

\begin{tabular}{cccccccc}
\hline \multirow{N}{*}{$N$} & $\boldsymbol{n}$ & HWHM & $\omega_{p l}$ & $\boldsymbol{D}$ & $\frac{\boldsymbol{H W H M}}{\omega_{p l}}$ & $\frac{H W H M}{D}$ & Bound \\
\hline 3 & 3 & 31.7 & 164 & $12,191.6$ & 0.19 & 0.0026 & $\mathrm{Y}$ \\
3 & 4 & 121.17 & 164 & 6857.8 & 0.74 & 0.0177 & $\mathrm{Y}$ \\
3 & 5 & 275.07 & 164 & 4389.0 & 1.68 & 0.0627 & $\mathrm{Y}$ \\
3 & 6 & 462.9 & 164 & 3047.9 & 2.82 & 0.1519 & $\mathrm{Y}$ \\
3 & 7 & 593.4 & 164 & 2239.3 & 3.62 & 0.2650 & $\mathrm{Y}$ \\
3 & 8 & 745.64 & 164 & 1714.4 & 4.55 & 0.4349 & $\mathrm{Y}$ \\
3 & 9 & 878.8 & 164 & 1354.6 & 5.36 & 0.6488 & $\mathrm{Y}$ \\
3 & 10 & 1045.8 & 164 & 1097.2 & 6.38 & 0.9531 & $\mathrm{Y}$ \\
3 & 11 & 1199.4 & 164 & 906.8 & 7.31 & 1.3226 & $\mathrm{~N}$ \\
\hline
\end{tabular}


Table 3. Cont

\begin{tabular}{cccccccc}
\hline $\boldsymbol{N}$ & $\boldsymbol{n}$ & HWHM & $\omega_{p l}$ & $\boldsymbol{D}$ & $\frac{H W H M}{\omega_{p l}}$ & $\frac{H W H M}{D}$ & Bound \\
\hline 3 & 12 & 1199.4 & 164 & 762.0 & 7.31 & 1.5740 & $\mathrm{~N}$ \\
10 & 3 & 97.5 & 299 & $12,191.6$ & 0.33 & 0.0080 & $\mathrm{Y}$ \\
10 & 4 & 279.5 & 299 & 6857.8 & 0.93 & 0.0408 & $\mathrm{Y}$ \\
10 & 5 & 514.6 & 299 & 4389.0 & 1.72 & 0.1172 & $\mathrm{Y}$ \\
10 & 6 & 816.5 & 299 & 3047.9 & 2.73 & 0.2679 & $\mathrm{Y}$ \\
10 & 7 & 932.0 & 299 & 2239.3 & 3.11 & 0.4162 & $\mathrm{Y}$ \\
10 & 8 & 1099.9 & 299 & 1714.4 & 3.67 & 0.6416 & $\mathrm{Y}$ \\
10 & 9 & 1404.7 & 299 & 1354.6 & 4.69 & 1.0370 & $\mathrm{~N}$ \\
10 & 10 & 1404.7 & 299 & 1097.2 & 4.69 & 1.2802 & $\mathrm{~N}$ \\
30 & 3 & 219.2 & 519 & $12,191.6$ & 0.42 & 0.0180 & $\mathrm{Y}$ \\
30 & 4 & 788.5 & 519 & 6857.8 & 1.52 & 0.1150 & $\mathrm{Y}$ \\
30 & 5 & 750.9 & 519 & 4389.0 & 1.45 & 0.1711 & $\mathrm{Y}$ \\
30 & 6 & 1490.1 & 519 & 3047.9 & 2.87 & 0.4889 & $\mathrm{Y}$ \\
30 & 7 & 1333.6 & 519 & 2239.3 & 2.57 & 0.5955 & $\mathrm{Y}$ \\
30 & 8 & 2407.0 & 519 & 1714.4 & 4.64 & 1.4040 & $\mathrm{~N}$ \\
\hline
\end{tabular}

Table 4. Widths vs. plasma frequency $\omega_{p l}$ and distance $D$ to the continuum for CONVPST with strong collisions as a function of the upper level principal quantum number $n$. All energies are in $\mathrm{cm}^{-1}$ and densities $N$ in $10^{17} \mathrm{e} / \mathrm{cm}^{3}$. The 'Bound' column indicates whether the state is considered bound $(\mathrm{Y})$ or $\operatorname{not}(\mathrm{N})$.

\begin{tabular}{cccccccc}
\hline $\boldsymbol{N}$ & $n$ & $\mathbf{H W H M}$ & $\boldsymbol{\omega}_{\boldsymbol{l} l}$ & $\boldsymbol{D}$ & $\frac{\boldsymbol{H W H M}}{\omega_{p l}}$ & $\frac{H W H M}{D}$ & Bound \\
\hline 3 & 3 & 11.5 & 164 & $12,191.6$ & 0.07 & 0.0009 & $\mathrm{Y}$ \\
3 & 4 & 121.9 & 164 & 6857.8 & 0.74 & 0.0178 & $\mathrm{Y}$ \\
3 & 5 & 284.7 & 164 & 4389.0 & 1.74 & 0.0649 & $\mathrm{Y}$ \\
3 & 6 & 517.8 & 164 & 3047.9 & 3.16 & 0.1699 & $\mathrm{Y}$ \\
3 & 7 & 526.6 & 164 & 2239.3 & 3.21 & 0.2351 & $\mathrm{Y}$ \\
3 & 8 & 537.1 & 164 & 1714.4 & 3.27 & 0.3133 & $\mathrm{Y}$ \\
3 & 9 & 602.2 & 164 & 1354.6 & 3.67 & 0.4445 & $\mathrm{Y}$ \\
3 & 10 & 993.6 & 164 & 1097.2 & 6.06 & 0.9055 & $\mathrm{Y}$ \\
3 & 11 & 764.5 & 164 & 906.8 & 4.66 & 0.8431 & $\mathrm{Y}$ \\
3 & 12 & 1077.7 & 164 & 762.0 & 6.57 & 1.4143 & $\mathrm{~N}$ \\
10 & 3 & 35.7 & 299 & $12,191.6$ & 0.12 & 0.0029 & $\mathrm{Y}$ \\
10 & 4 & 262.6 & 299 & 6857.8 & 0.88 & 0.0383 & $\mathrm{Y}$ \\
10 & 5 & 463.7 & 299 & 4389.0 & 1.55 & 0.1057 & $\mathrm{Y}$ \\
10 & 6 & 855.1 & 299 & 3047.9 & 2.86 & 0.2806 & $\mathrm{Y}$ \\
10 & 7 & 593.7 & 299 & 2239.3 & 1.98 & 0.2651 & $\mathrm{Y}$ \\
10 & 8 & 877.5 & 299 & 1714.4 & 2.93 & 0.5118 & $\mathrm{Y}$ \\
10 & 9 & 1056.8 & 299 & 1354.6 & 3.53 & 0.7801 & $\mathrm{Y}$ \\
10 & 10 & 1431.0 & 299 & 1097.2 & 4.78 & 1.3042 & $\mathrm{~N}$ \\
30 & 3 & 82.9 & 519 & $12,191.6$ & 0.16 & 0.0068 & $\mathrm{Y}$ \\
30 & 4 & 437.5 & 519 & 6857.8 & 0.84 & 0.0638 & $\mathrm{Y}$ \\
30 & 5 & 497.2 & 519 & 4389.0 & 0.96 & 0.1133 & $\mathrm{Y}$ \\
30 & 6 & 876.5 & 519 & 3047.9 & 1.69 & 0.2879 & $\mathrm{Y}$ \\
30 & 7 & 1174.9 & 519 & 2239.3 & 2.26 & 0.5247 & $\mathrm{Y}$ \\
30 & 8 & 2466.3 & 519 & 1714.4 & 4.75 & 1.4386 & $\mathrm{~N}$ \\
\hline & & & & & & & \\
\end{tabular}

It should be clear from these tables that the widths for the higher series members significantly exceed the plasma frequency, hence a frequency-independent collision operator, as in the impact approximation used for CONVST/PST, may be inadequate. It should be noted that the plasma frequency should be compared to the electron impact width (i.e., without ions). Thus, the width without ions should in principle be used, however this is not done.

Figure 7 illustrates the effect of the Lewis cutoff towards the series limit; in addition to accounting for incomplete collisions, the results with Lewis cutoff also employed the Inglis-Teller cutoff, resulting 
in fewer bound lines. As a result the wings are significantly suppressed compared to the calculations without the Lewis cutoff, both for CONVST and CONVPST. Strong collisions were included in all CONV runs in Figure 7.

\section{Balmer(bound-bound) series at the intermediate density beyond H-gamma}

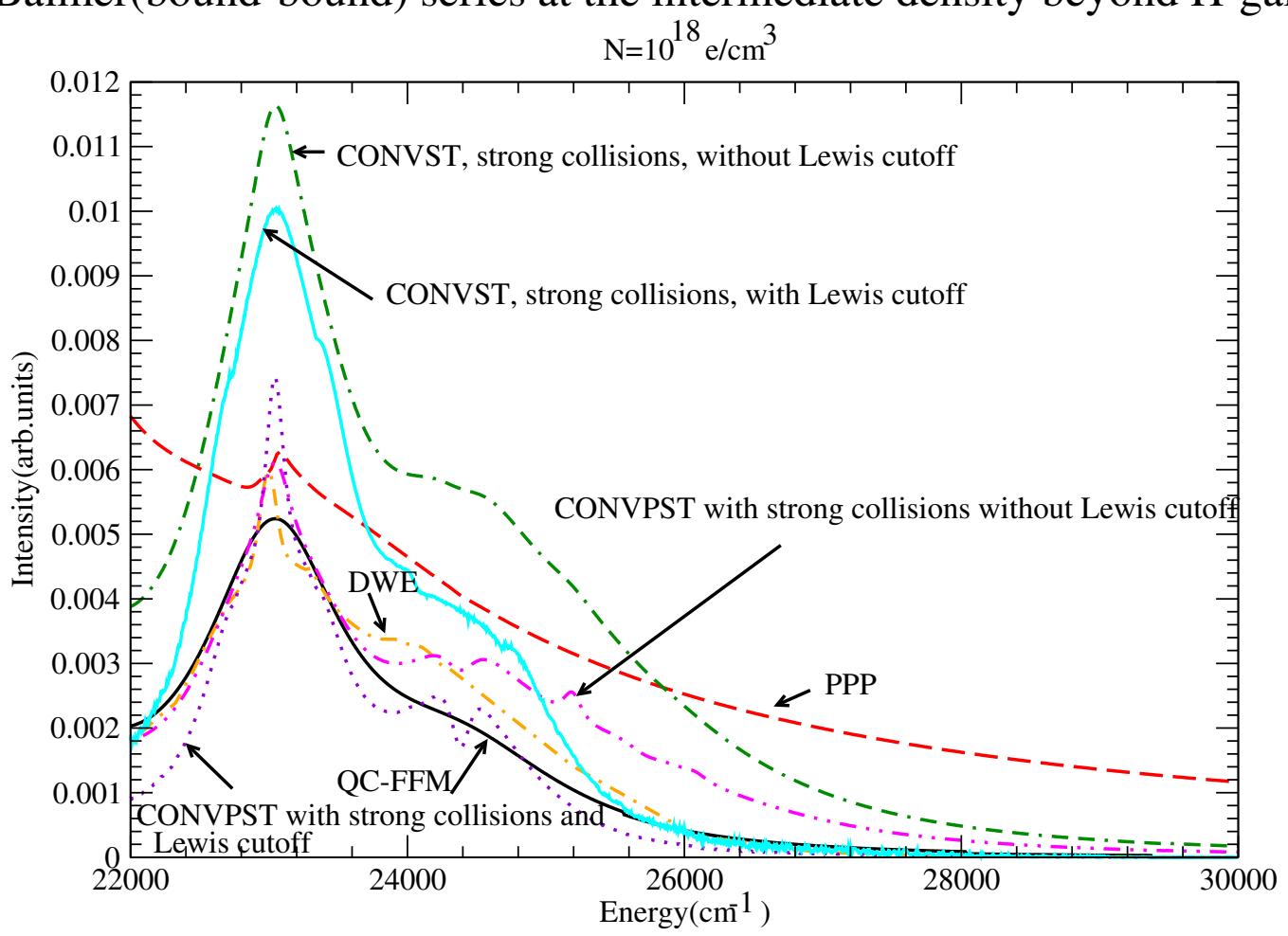

Figure 7. Balmer Series limit region with and without the Lewis cutoff for the intermediate density. Shown are PPP (red dashed), QC-FFM (back solid), DWE (orange dash-dotted), CONVST without Lewis cutoff (green dot-double dashed), CONVST with Lewis cutoff (cyan solid), CONVPST without Lewis cutoff (magenta dash-double dotted) and CONVPST with Lewis cutoff (violet dotted). All CONV runs include strong collisions.

\subsection{Remarks}

Overall, we expected a good agreement between the codes, as the line profiles are determined by quasistatic ions and (except for QC-FFM, which treats electrons via the FFM) impact electrons, with differences expected for the higher members of the series, as strong collisions and penetration, which are not treated in the same way in all codes should result in some differentiation.

As specified, and in order to focus on all series members more or less equally, the codes were modified for the purposes of this comparison so that the Boltzmann factor for the upper level population is taken to be 1 for all lines (i.e., the relative intensity is $\left|\mathbf{d}_{\alpha \beta}\right|^{2}$, where $\mathbf{d}$ is the dipole matrix element and $\alpha, \beta$ are upper and lower level states respectively). In addition, the $\omega^{4}$ factor was also neglected. With regard to normalization, peak height of the entire series (i.e., $H_{\alpha}$ peak) was taken to be 1 .

\section{Bound-Bound Transitions: Results}

In the comparison we first took into account bound-bound transitions exclusively. That is, the codes estimate an upper principal quantum number $n^{*}$, as explained above and compute the entire series up till that quantum number $n^{*}$ as shown in Table 5. 
Table 5. $n^{*}$ for the different codes as a function of the electron density $N$, expressed in units of $10^{17} \mathrm{e} / \mathrm{cm}^{3}$.

\begin{tabular}{cccccccc}
\hline $\boldsymbol{N}$ & QC-FFM & PPP & DWE & CONVST-Weak & CONVST & CONVPST-Weak & CONVPST \\
\hline 3 & 6 & 15 & 7 & 13 & 10 & 13 & 11 \\
10 & 6 & 15 & 7 & 8 & 8 & 9 & 9 \\
30 & 5 & 15 & 6 & 8 & 7 & 9 & 7 \\
\hline
\end{tabular}

In CONVST/PST, for the lowest density $n^{*}$ for weak collisions only was determined because at the next higher $n$, the wavefunction extent is larger than the interatomic spacing as discussed above. When strong collisions are neglected, this occurs before the HWHM becomes larger than the distance to the continuum. For the highest density in CONVST $n^{*}$ is determined from the condition that for the next higher $n$, the minimum impact parameter is larger than the maximum impact parameter and as a result all collisions are "strong". Note that in principle for high $n$, small differences in the widths by the different codes could result in high $n$ states being classified as either bound/observable (for the codes producing the smaller widths) or free/unobservable (for the codes producing the larger widths). As a result lines may be included or left out, with resulting significant differences towards the series limit; however the main differences arise from the different ways in the choice of $n^{*}$, specifically that QC-FFM uses the Inglis-Teller criterion (i.e., stop the series when the HWHM exceeds the distance to the next level), while CONVST/PST continues until the HWHM exceeds the distance to the continuum.

\subsection{Lowest Density}

Figure 8 displays a view of the entire series. This is dominated intensity-wise by the $H_{\alpha}$ line, as expected, while lines appear not to overlap. This is correct nearly all the way to the limit, as illustrated in Figure 9. The last clearly visible line in all codes is $H_{\delta}(n=6)$, with CONVPST and DWE showing the characteristic dip. Interestingly enough good agreement is obtained for all codes, except CONVST, i.e., a standard calculation with a strong collision term corresponding to the assumption that $\left\{1-S_{a}(\rho, v) S_{b}^{*}(\rho, v)\right\}=1$. In contrast, CONVPST, with no strong collision which accounts for penetration gives significantly narrower shapes and agrees with codes that do not account for penetration. CONVST with no strong collision term (weak collisions only) also agrees with the other codes; however, predictably for the higher lines, strong collisions dominate and CONVST-weak only is noisy, as the weak-only widths are very small. Especially for the higher density runs, where for many codes the lines are not discernible, CONVST-weak is a useful indicator both for the line positions and for their width that is not due to strong (in the ST sense, i.e., without account for penetration) collisions.

Looking in more detail in Figure $10, H_{\alpha}$, which among the lines affected by electron broadening, would be least affected by strong collisions due to its smaller polarizability, provides a measure of the differences in the handling of the strong collision term: If we use QC-FFM (solid line) as a separator, we have CONVST with the strong collision term and PPP displaying a distinctly wider line, while the remaining codes produce narrower lines. This indicates that the strong collision term adopted by CONVST $\left\{1-S_{a}(\rho, v) S_{b}^{*}(\rho, v)\right\}=1$ and to a lesser extent PPP seems to be significantly larger than all other codes. We also note a shift, particularly for DWE; however, shifts are out of scope of the present comparisons and which will be discussed here. Note that for $\mathrm{H}$-alpha, while the strong collisions account for $50-73 \%$ of the total electronic broadening for ST, they only account for $4-7 \%$ of the electronic broadening in PST; concequently the PST is significantly narrower for $H-\alpha$, which has the strongest electronic component in the series and hence differences in electron broadening are more pronounced. We note that the QC model, specifically derived for $\Delta n \gg 1$ transitions, is less suited for $\Delta n=1$ lines, as is the case with $H_{\alpha}$. Nevertheless, here it agrees remarkably well with DWE and CONVPST. 
Entire (bound-bound) series at the lowest density

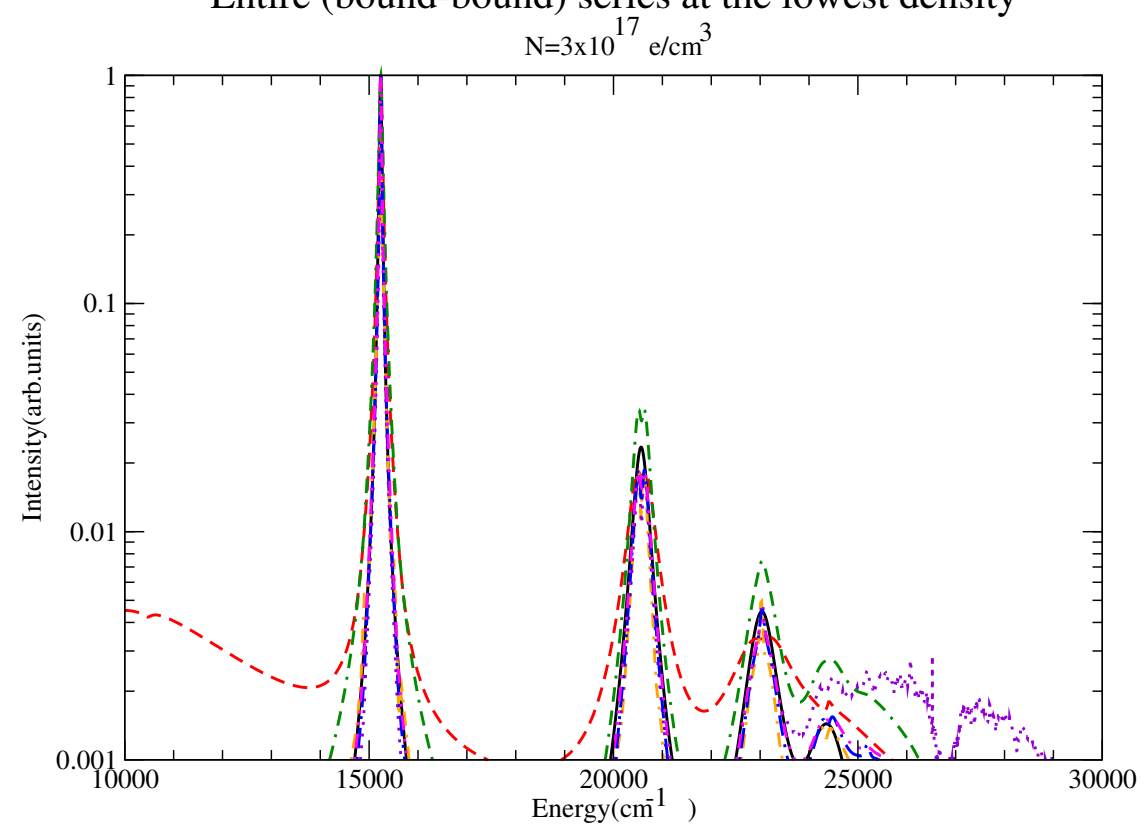

Figure 8. The entire series for the lowest density considered for QC-FFM (solid black), PPP (dashed red), DWE (dash-dotted orange), CONVST without strong collisions (dotted violet), CONVST with strong collisions (dot-double dashed green), CONVPST without strong collisions (long dash-dotted blue) and CONVPST with strong collisions (dash-double dotted magenta).

Balmer (bound-bound) series at the lowest density: H-gamma and beyond

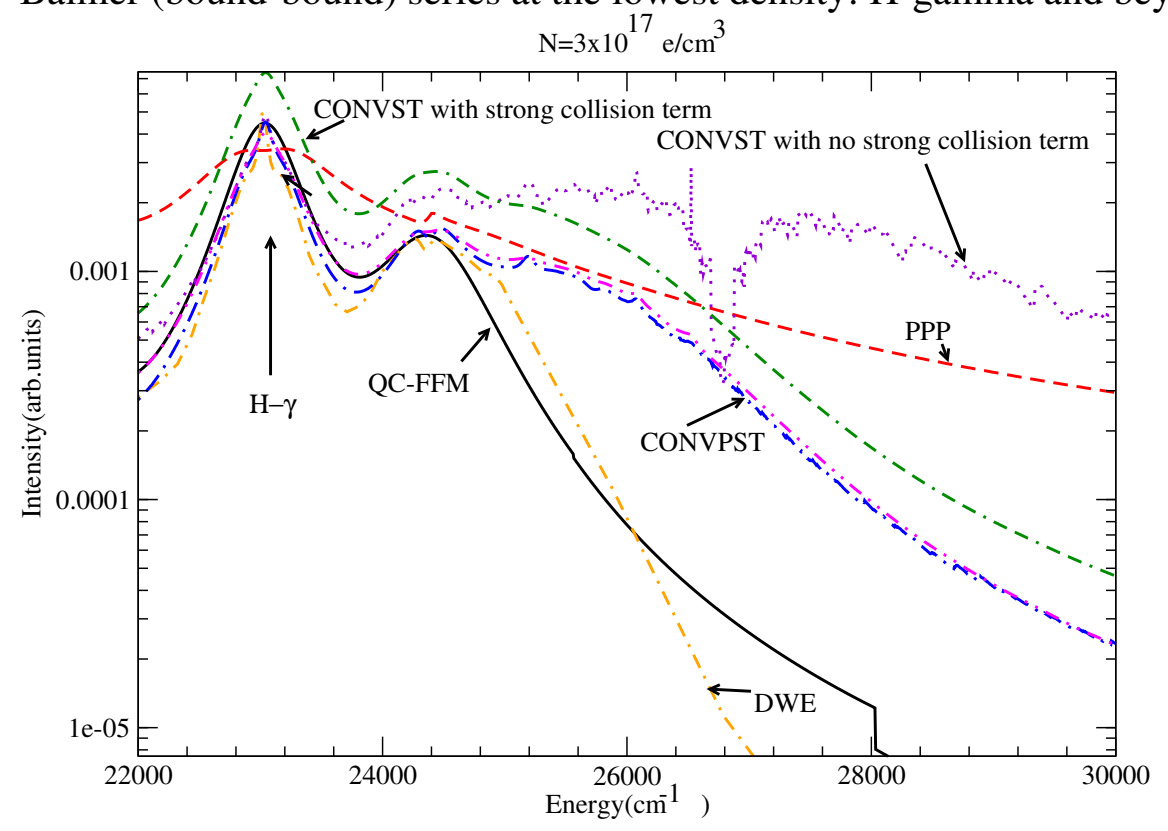

Figure 9. The series from $H_{\gamma}$ to the continuum at the lowest density showing fairly small overlap. Shown are QC-FFM (solid), PPP (dashed), DWE (dash-dotted), CONVST without strong collisions (dotted), CONVST with strong collisions (dot-double dashed), CONVPST without strong collisions (long dash-dotted) and CONVPST with strong collisions (dash-double dotted).

Looking at the series from $H_{\beta}$ to the series limit in Figure 11 we again note the same trends, with DWE, CONVPST and QC-FFM agreeing well except towards the series limit, with PPP and CONVST being significantly wider, probably due to the collision operator and the inclusion of more states with 
overestimated wing intensities, while CONVST without strong collisions is narrower and becomes noisy towards the series limit. This is understandable, because DWE includes up to $n^{*}=7$, while CONVPST includes more states due to the chosen cutoff.

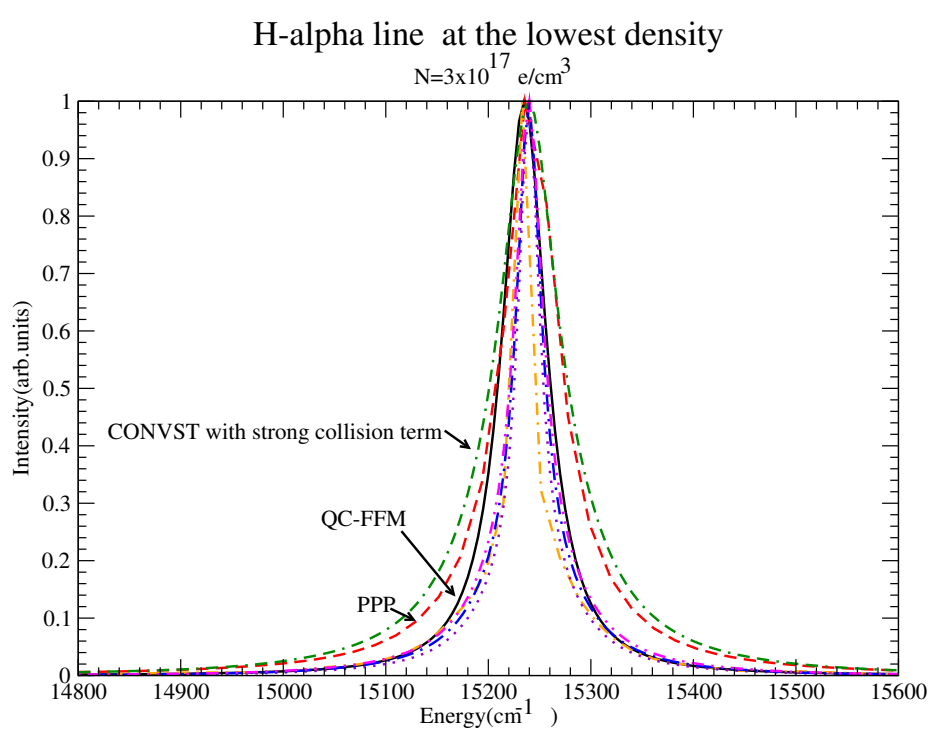

Figure 10. $H_{\alpha}$ at the lowest density. Shown are QC-FFM (solid), PPP (dashed), DWE (dash-dotted), CONVST without strong collisions (dotted), CONVST with strong collisions (dot-double dashed), CONVPST without strong collisions (long dash-dotted) and CONVPST with strong collisions (dash-double dotted). Note the good agreement betwen DWE, CONVPST and QC-FFM, while according to most codes, the strong collision term in CONVST appears to significantly overestimate the width, while ignoring it significantly underestimates it. PPP also appears to overestimate the width, as expected due to the GBK operator without frequency dependence.

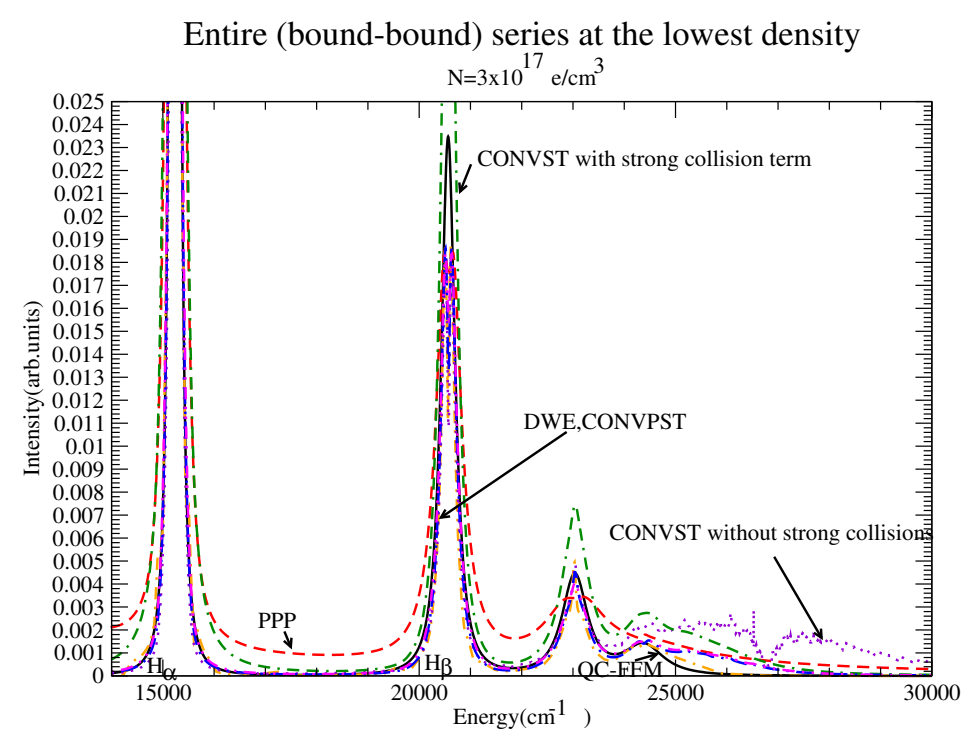

Figure 11. Comparision of line widths. Shown are QC-FFM (solid), PPP (dashed), DWE (dash-dotted), CONVST without strong collisions (dotted), CONVST with strong collisions (dot-double dashed), CONVPST without strong collisions (long dash-dotted) and CONVPST with strong collisions (dash-double dotted). It is clear that PPP is widest, probably due to the GBK operator; among the remaining codes it is also clear that the strong collision term adopted by CONVST is too large.

We also note that QC-FFM does not display the characteristic dip in the $H_{\beta}$ center, but this is a known and expected artifact of QC, with little effect on the widths. 
In Figure 12 the series limit region is displayed. Only CONVPST apart from CONVST-weak shows some structure, a trend that was already visible from Figure 9 where DWE and CONVPST differ significantly after the $H_{\delta}$ line, as does QC-FFM. Note that up until $H_{\delta}$, DWE and CONVPST were in very good agreement throughout and QC-FFM was also very close. This is attributable to CONV having a larger cutoff principal quantum number $n^{*}$.

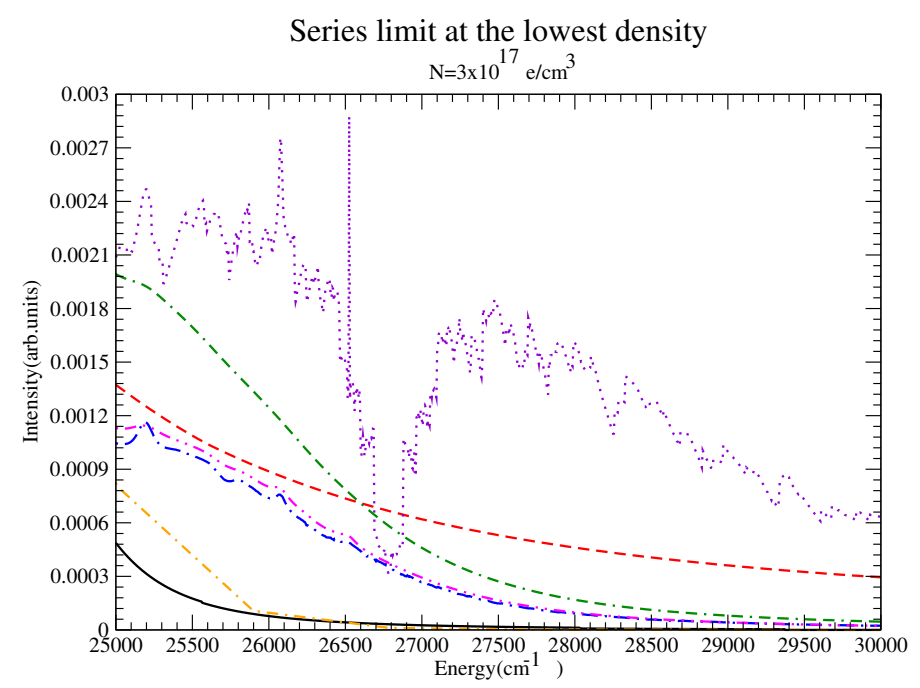

Figure 12. Comparision of line widths. Shown are QC-FFM (solid), PPP (dashed), DWE (dash-dotted), CONVST without strong collisions (dotted), CONVST with strong collisions (dot-double dashed), CONVPST without strong collisions (long dash-dotted) and CONVPST with strong collisions (dash-double dotted).

To investigate the effect of the Inglis-Teller and Lewis cutoffs, Figure 13 shows the same region as Figure 9 from $H_{\gamma}$ to the continuum for CONV with strong collisions using both the Inglis-Teller and Lewis cutoffs. This results in a significant reduction of the blue wing and a much better agreement on the blue wing with DWE and QC-FFM.

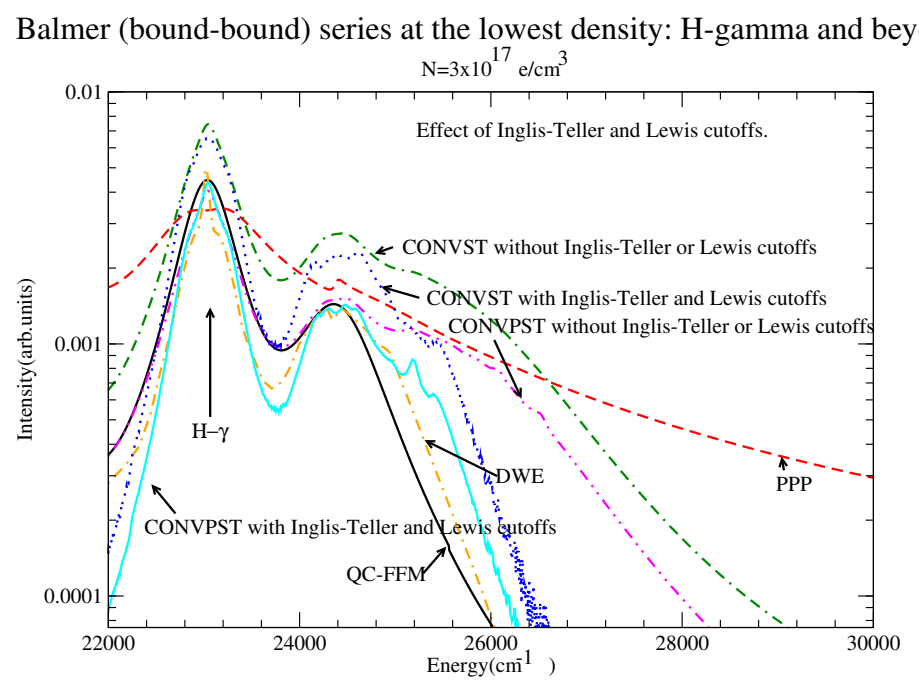

Figure 13. The series from $H_{\gamma}$ to the continuum at the lowest density. Shown are QC-FFM (solid black), PPP (dashed red), DWE (dash-dotted), CONVST without the Inglis-Teller or Lewis cutoffs (dot-double dashed green), CONVST with the Inglis-Teller and Lewis cutoffs (dotted blue) CONVPST with the Inglis-Teller and Lewis cutoffs (solid cyan) and CONVPST without the Inglis-Teller or Lewis cutoffs (magenta dash-double dotted). Strong collisions are included in all CONV runs. 


\subsection{Intermediate Density}

Figure 14 displays a view of the entire series, again dominated intensitywise by the $H_{\alpha}$ line shown in detail in Figure 15, as expected, while some overlap towards the series limit is visible. This is more clearly seen in Figure 16.

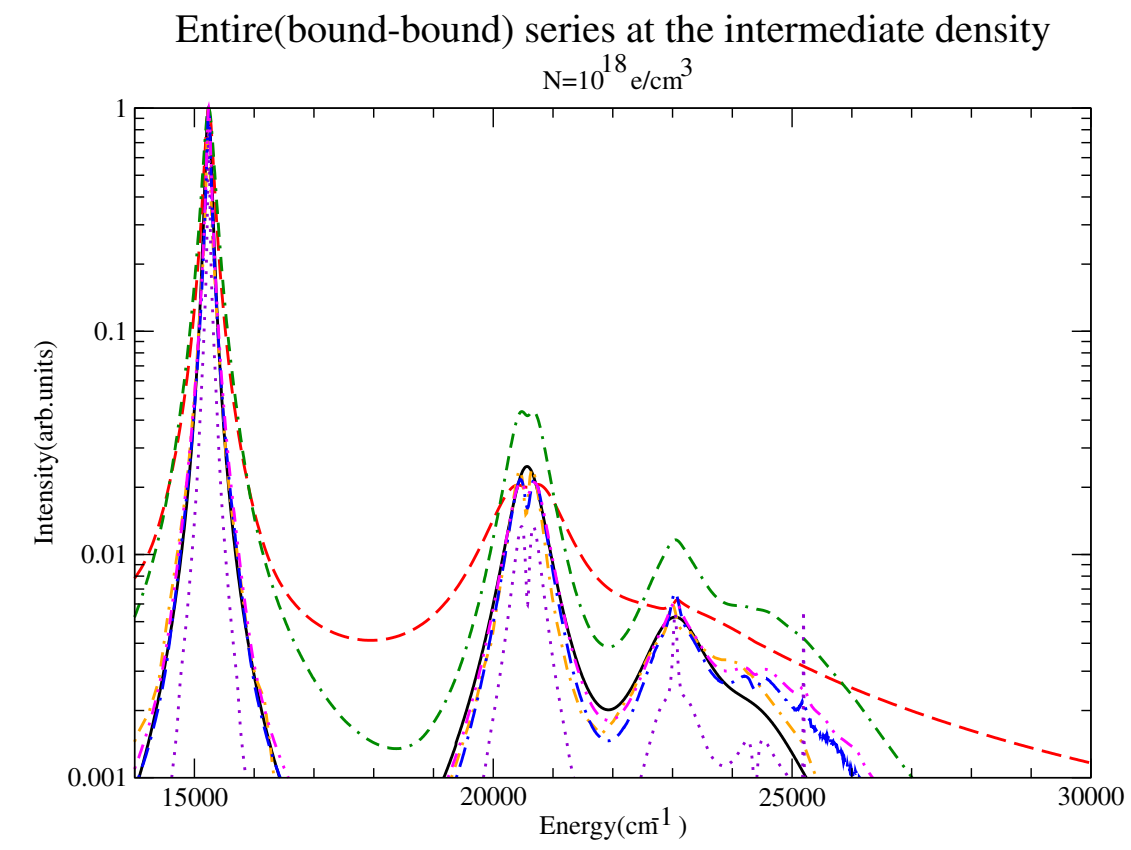

Figure 14. The entire series for the intermediate density considered for QC-FFM (solid black), PPP (dashed red), DWE (dash-dotted orange), CONVST without strong collisions (dotted violet), CONVST with strong collisions (dot-double dashed green), CONVPST without strong collisions (long dash-dotted blue) and CONVPST with strong collisions (dash-double dotted magenta).

$\mathrm{H}$-alpha at the intermediate density

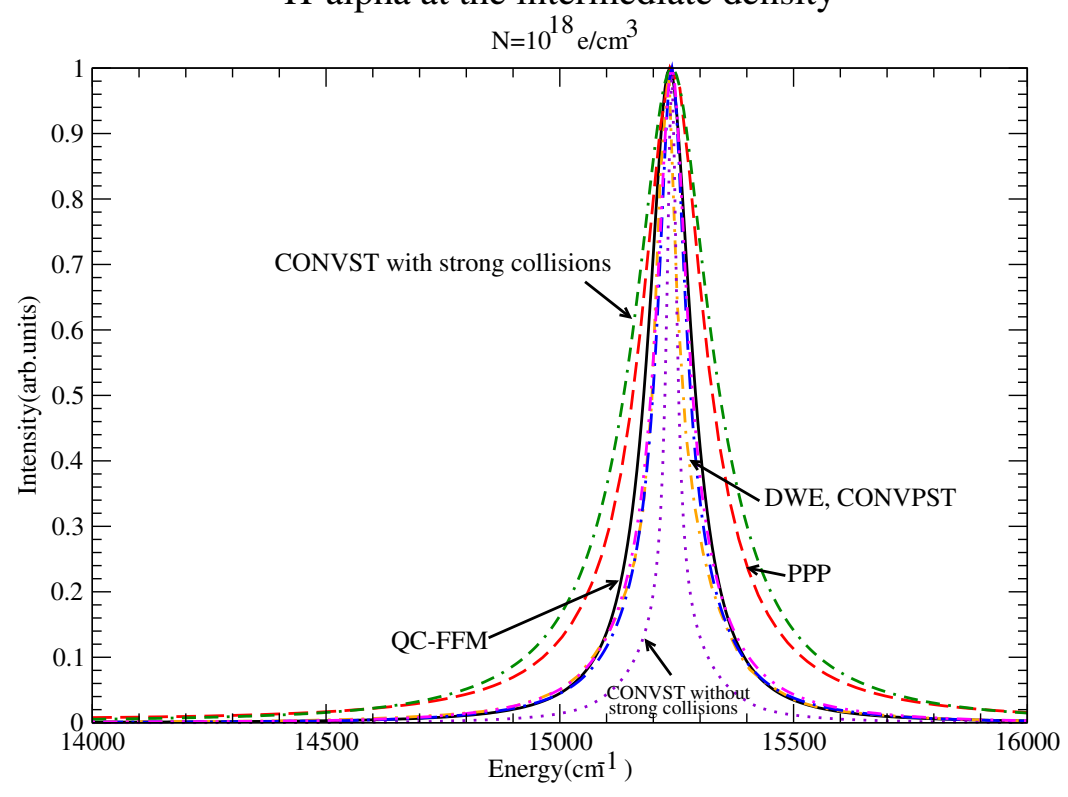

Figure 15. $H_{\alpha}$ at the intermediate density showing a very good agreement between QC-FFM, CONVPST and DWE. Shown are QC-FFM (solid), PPP (dashed), DWE (dash-dotted), CONVST without strong collisions (dotted), CONVST with strong collisions (dot-double dashed), CONVPST without strong collisions (long dash-dotted) and CONVPST with strong collisions (dash-double dotted). 
DWE, CONVPST entire series at the intermediate density

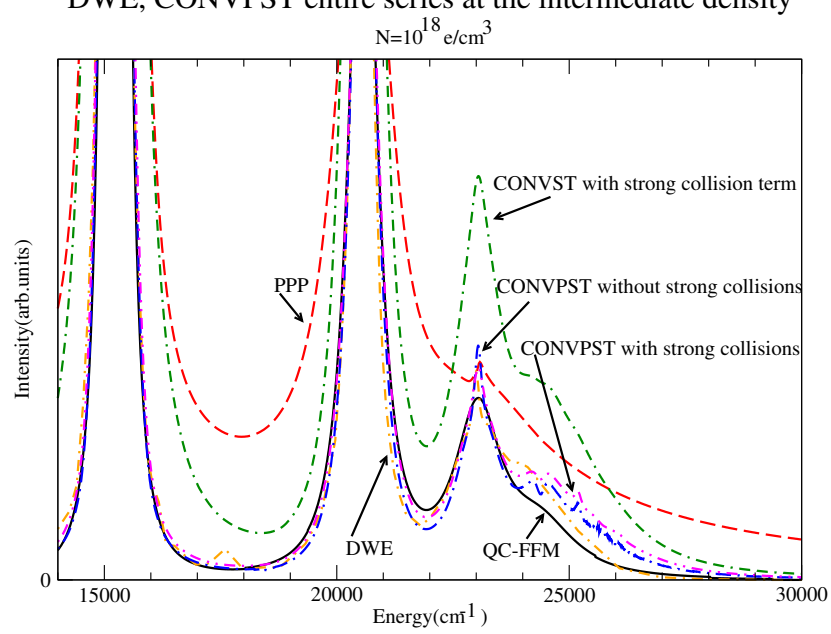

Figure 16. The series from $H_{\gamma}$ to the continuum for the intermediate density considered for DWE (dash-dotted orange) and CONVPST without (long dash-dotted blue) and with strong collisions (dash-double dotted magenta). For comparison PPP (red dashed) and QC-FFM (solid black) are also shown.

Figures 4 and 16 show the high energy part of the series. Interestingly enough only CONVPST shows the $H_{\delta}$ dip as well as an $H_{\epsilon}$ peak; although these lines are included in , for instance, DWE, only a broad wing is visible in DWE. Hence again fairly small differences in broadening can be decisive in whether a line is visible or not. Note that DWE and CONVPST agree quite well up until $H_{\gamma}$, with DWE only displaying, at best, a shoulder of $H_{\delta}$.

We also show the region close to the series limit in Figure 17: Once again, the general trends that appeared in the lowest density appear here as well: Only CONVPST, apart from CONVST-weak shows any structure. In addition, the codes differ significanty in their blue "wings", probably due to the different number of states included and the handling of the wings and frequency dependence of the collision operators. Furthermore, CONVPST and CONVPST-weak display some small, but clear differences, indicating the growing importance of penetrating strong collisions. The step-wise structure seen in QC-FFM is an artifact of the calculation, as QC-FFM calculates the wings up till $|\Delta \omega| \approx 10 H W H M$.

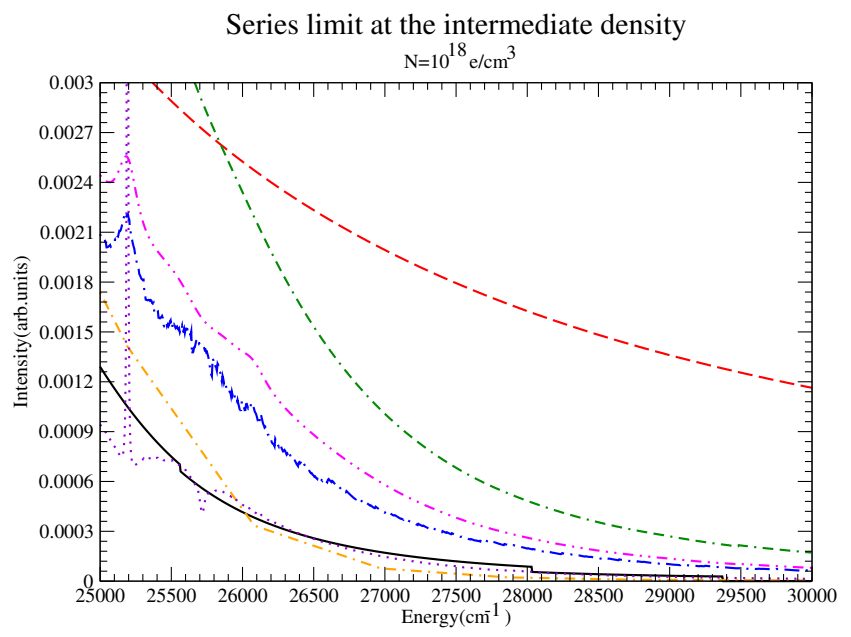

Figure 17. Comparision of line widths. Shown are QC-FFM (solid), PPP (dashed), DWE (dash-dotted), CONVST without strong collisions (dotted), CONVST with strong collisions (dot-double dashed), CONVPST without strong collisions (long dash-dotted) and CONVPST with strong collisions (dash-double dotted). 
As already shown in Figure 7, inclusion of the Lewis cutoff and use of the Inglis-Teller bound state criterion significantly improves the blue wing agreement of CONV with QC-FFM and DWE.

\subsection{Highest Density}

As expected, the highest density exhibits more overlap, as in Figure 18.

Entire (bound-bound) series at the highest density

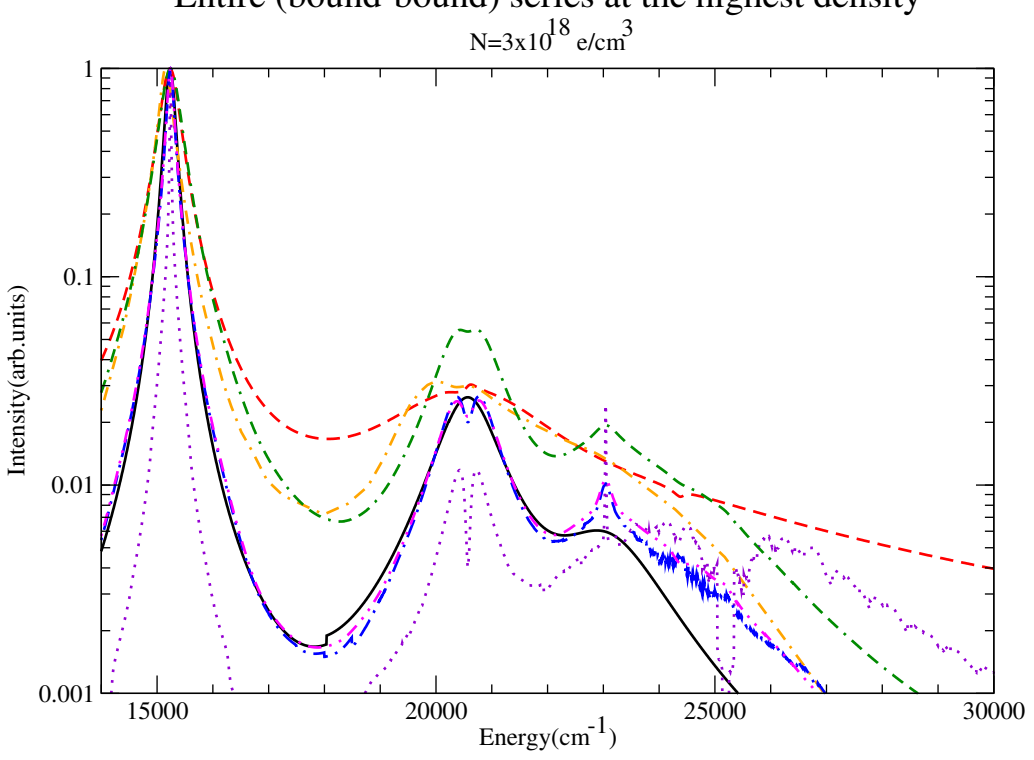

Figure 18. The entire series at the highest density. Shown are QC-FFM (solid), PPP (dashed), DWE (dash-dotted), CONVST without strong collisions (dotted), CONVST with strong collisions (dot-double dashed), CONVPST without strong collisions (long dash-dotted) and CONVPST with strong collisions (dash-double dotted).

Figure 19 focuses on the region including and beyond $H_{\gamma}$, where the codes differ significantly.

Balmer (bound-bound) series at the highest density: H-gamma and beyond

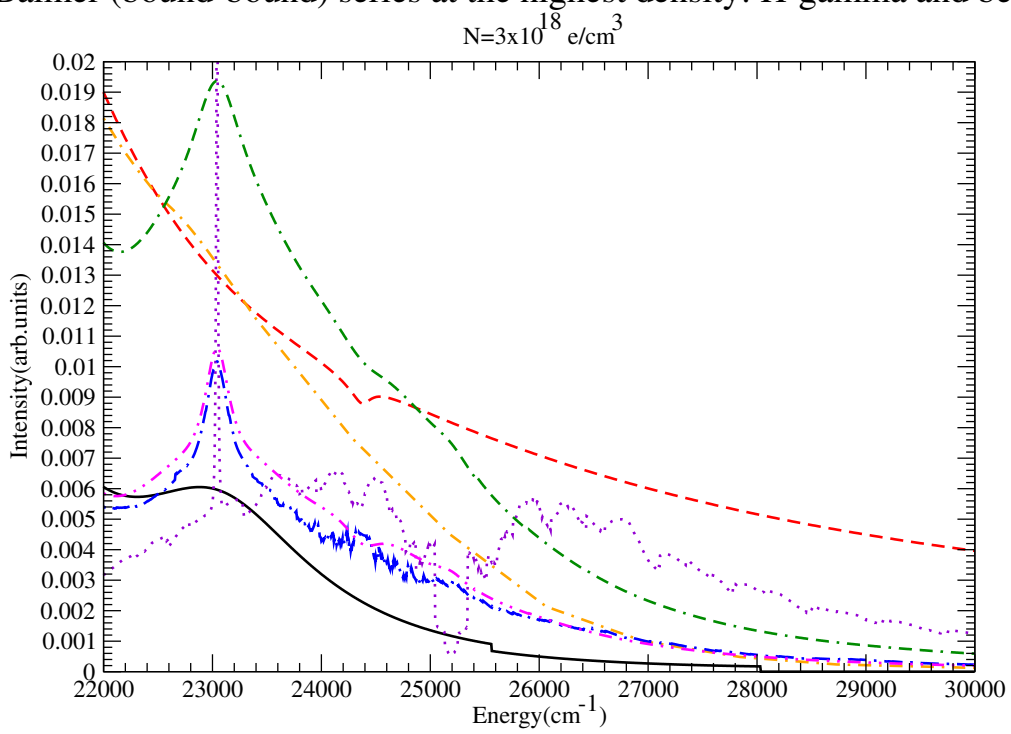

Figure 19. The region including and beyond $H_{\gamma}$ at the highest density. Shown are QC-FFM (solid), PPP (dashed), DWE (dash-dotted), CONVST without strong collisions (dotted), CONVST with strong collisions (dot-double dashed), CONVPST without strong collisions (long dash-dotted) and CONVPST with strong collisions (dash-double dotted). 
Figure 20 displays the region around $H_{\alpha}$, where QC-FFM and CONVPST show a good agreement, with visible differences from other codes. This agreement between these codes extends up until $H_{\gamma}$, as shown in Figure 21.

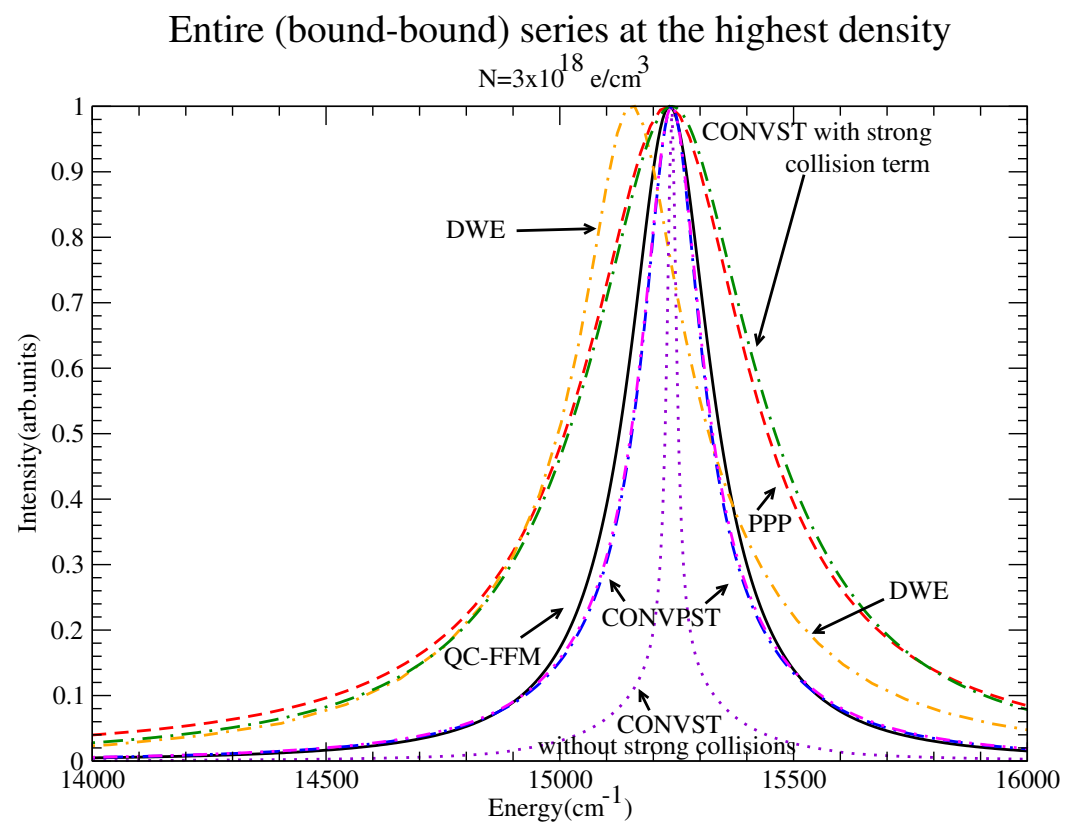

Figure 20. $H_{\alpha}$ at the highest density showing a very good agreement between QC-FFM and CONVPST. Shown are QC-FFM (solid), PPP (dashed), DWE (dash-dotted), CONVST without strong collisions (dotted), CONVST with strong collisions (dot-double dashed), CONVPST without strong collisions (long dash-dotted) and CONVPST with strong collisions (dash-double dotted).

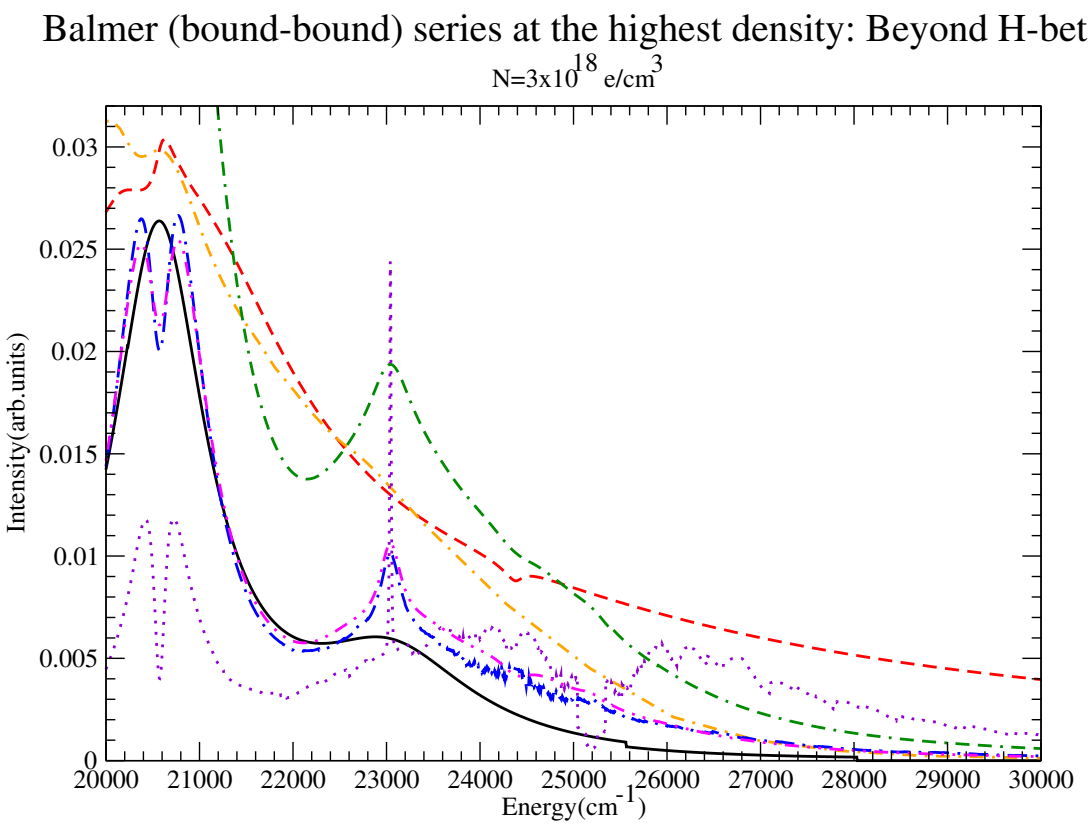

Figure 21. The region including $H_{\beta}$ and beyond at the highest density showing a very good agreement between QC-FFM and CONVPST up until $H_{\gamma}$. Shown are QC-FFM (solid), PPP (dashed), DWE (dash-dotted), CONVST without strong collisions (dotted), CONVST with strong collisions (dot-double dashed), CONVPST without strong collisions (long dash-dotted) and CONVPST with strong collisions (dash-double dotted). 
In Figure 22 the region around the series limit is displayed, with only CONVPST-weak showing any structure apart from CONVST-weak and codes differing widely.

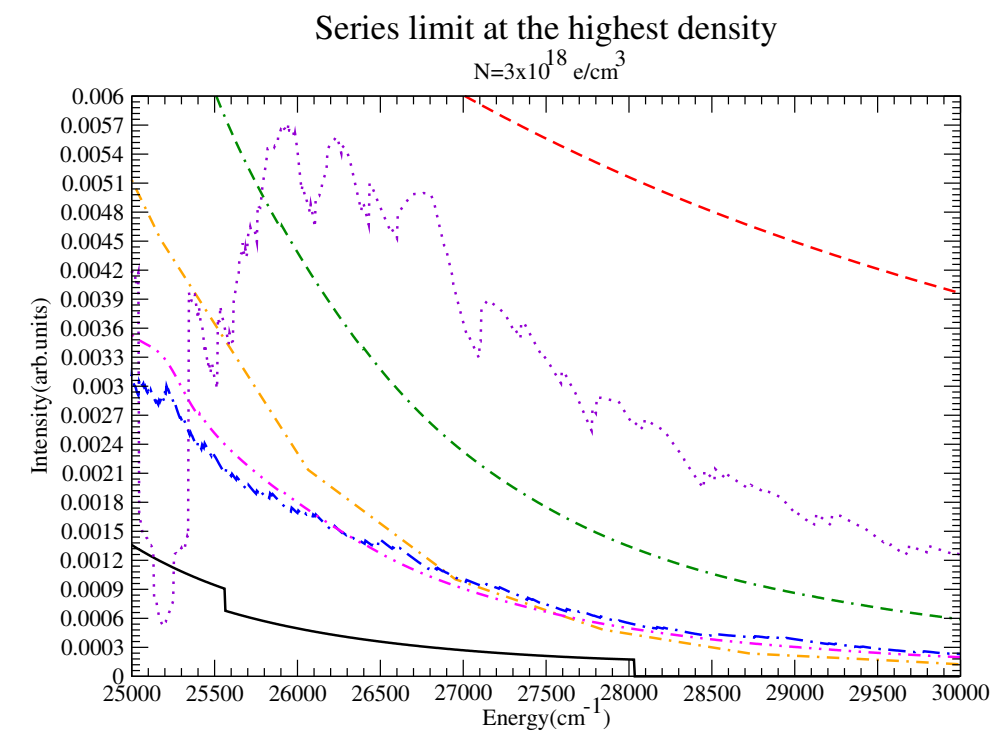

Figure 22. The region near the series limit at the highest density. Shown are QC-FFM (solid), PPP (dashed), DWE (dash-dotted), CONVST without strong collisions (dotted), CONVST with strong collisions (dot-double dashed), CONVPST without strong collisions (long dash-dotted) and CONVPST with strong collisions (dash-double dotted).

To understand these differences, we show Figure 23, which is essentially Figure 18, this time without the CONV-weak collisions only results, but with extra CONVST/PST runs with (a) the Inglis-Teller cutoff and (b) the Lewis cutoff. It is seen that the blue wings show a significantly better agreement with QC-FFM and DWE. Specifically $H_{\gamma}$ disappears when the Inglis-Teller cutoff is used, while the wings between $H_{\alpha}$ and $H_{\beta}$ (as well as the blue wings) are suppressed significantly.

Entire (bound-bound) series at the highest density

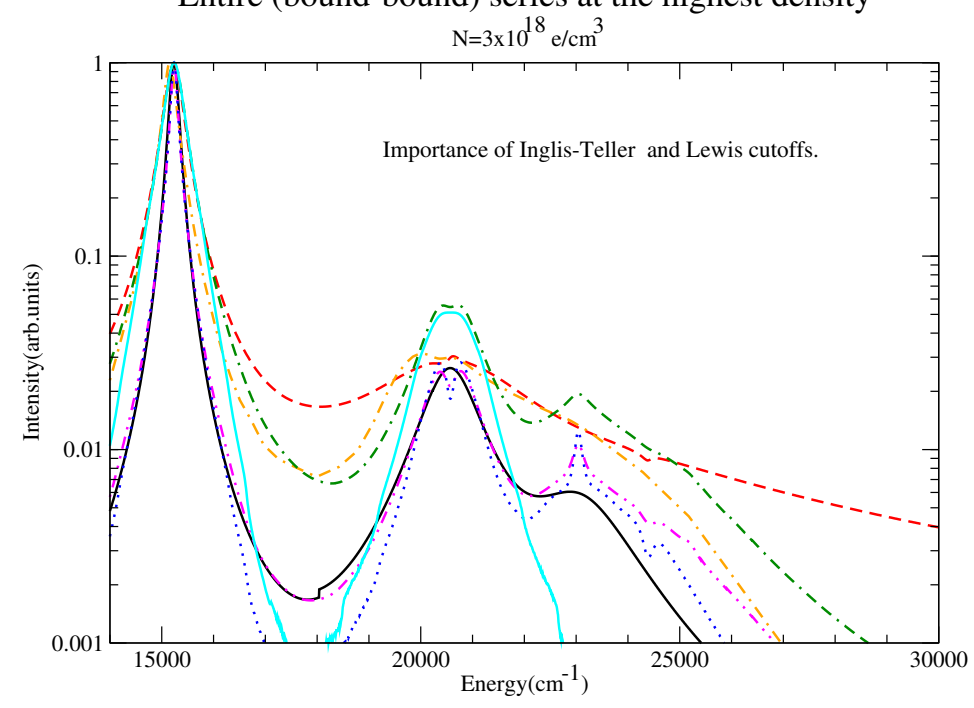

Figure 23. The entire series at the highest density. Shown are QC-FFM (black solid), PPP (red dashed), DWE (orange dash-dotted), CONVST without Inglis-Teller or Lewis cutoff (green dot-double dashed), CONVST with Inglis-Teller and Lewis cutoff (cyan solid), CONVPST without Inglis-Teller or Lewis cutoff (magenta dash-double dotted) and CONVPST with Inglis-Teller and Lewis cutoff (blue dotted). All CONVST/PST runs include strong collisions. 


\section{Addition of Free-Bound Transitions: Results}

\subsection{General Remarks}

As specified by the problem statement, free-bound contributions to the spectrum were also requested. For this reason, transitions from higher principal quantum numbers than those considered "still bound" were added. This would seem to simply add a very wide, thus kind of flat "baseline" with decreasing contributions because of the $\left|\mathbf{d}_{\alpha \beta}\right|^{2}$, intensity factor. However, the spacing between consecutive upper level principal quantum numbers becomes smaller and exactly compensates the decrease of the matrix elements, since $n^{-2}-(n+1)^{-2} \approx n^{-3}$. Thus, the net result is expected to be the addition of a very nearly constant "baseline" if the width is constant. Indeed, for the Lyman series considered in the previous workshop, it was shown that exact calculations reproduce the flat QC-FFM line reasonably well in the region between the Inglis-Teller limit and the last $(n=10$ in the Lyman series computation) level. In the present comparison, a key question is whether the wings from lower-lying lines complicate this expectation due to the higher intensity of these lower-lying lines.

Here we include lines that are not bound (e.g., up to $n=20$ for CONVST $/$ PST). These extra lines are very close, i.e., HWHM $>$ Distance to the Continuum $\gg$ distance between levels $=n^{-2}-(n+1)^{-2} \approx$ $n^{-3}$. Such lines are quite flat. Far from the continuum, the series profile does not change much.

CONVST/PST and QC-FFM participated in this comparison for all cases. DWE contributed for the intermediate case. QC-FFM is quite flat. Indeed the high- $n$ lines are flat; however, the profile is still dominated by the (higher intensity), lower $n$-line wings for CONVST/PST, which, as discussed did not employ frequency-dependent collision operator. By accounting for all levels up to $n=15$, PPP partially deals with this case. The remarks made for CONVST/PST regarding the use of a frequency-independent operator also apply for PPP. As shown in Table 2, in ST, even for the lowest density considered, all collisions are "strong" for $n>12$, as the minimum impact parameter exceeds the maximum impact parameter. In this respect, the only critical ST electron impact aspect is the strong collision estimate. This is not the case for PST where there is no minimum impact parameter.

\subsection{The Region far from the Continuum}

Clearly the addition of FB will ony affect the portion of the spectrum close to the series limit. Indeed, $H_{\alpha}$ is easily seen to be unaffected in Figure 24 for the intermediate density.

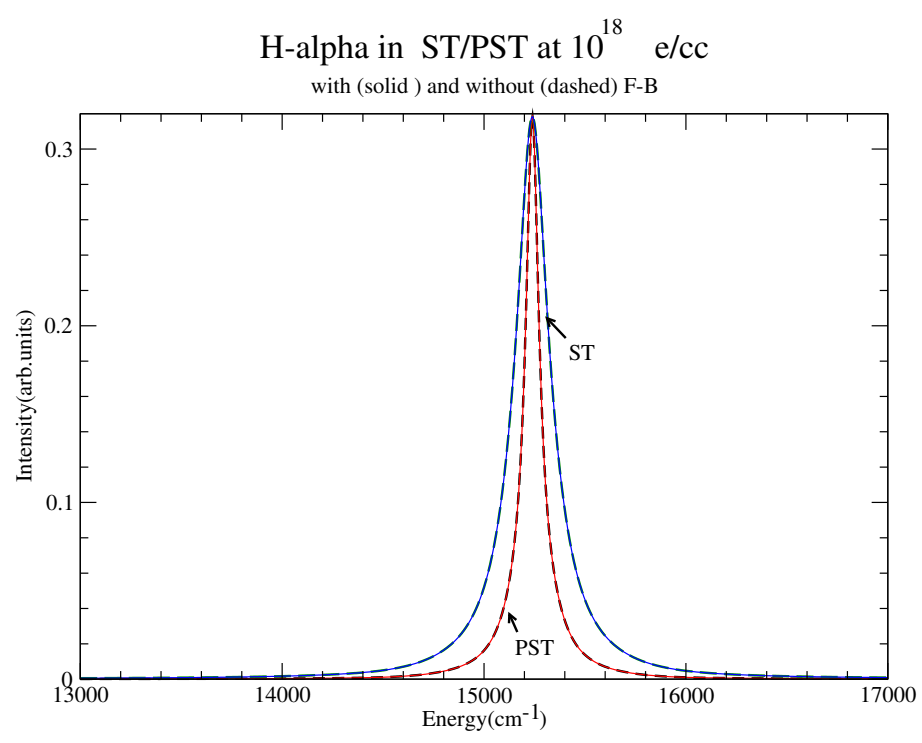

Figure 24. BB + FB vs. BB comparison for the $H_{\alpha}$ line at the intermediate density. Shown CONVST for BB + FB (blue solid) and CONVST for BB + FB (red solid) and the corresponding BB only results (dashed), which are identical. 
Figures 25 and 26 display and contrast the Bound-Bound (BB) and Bound-Bound plus Free-Bound $(\mathrm{BB}+\mathrm{FB})$ spectra far from the continuum for the lowest and intermediate densities.

$\mathrm{FB}+\mathrm{BB}$ vs $\mathrm{BB}$ for $\mathrm{N}=3 \times 10^{17} \mathrm{e} / \mathrm{cm}^{3}$

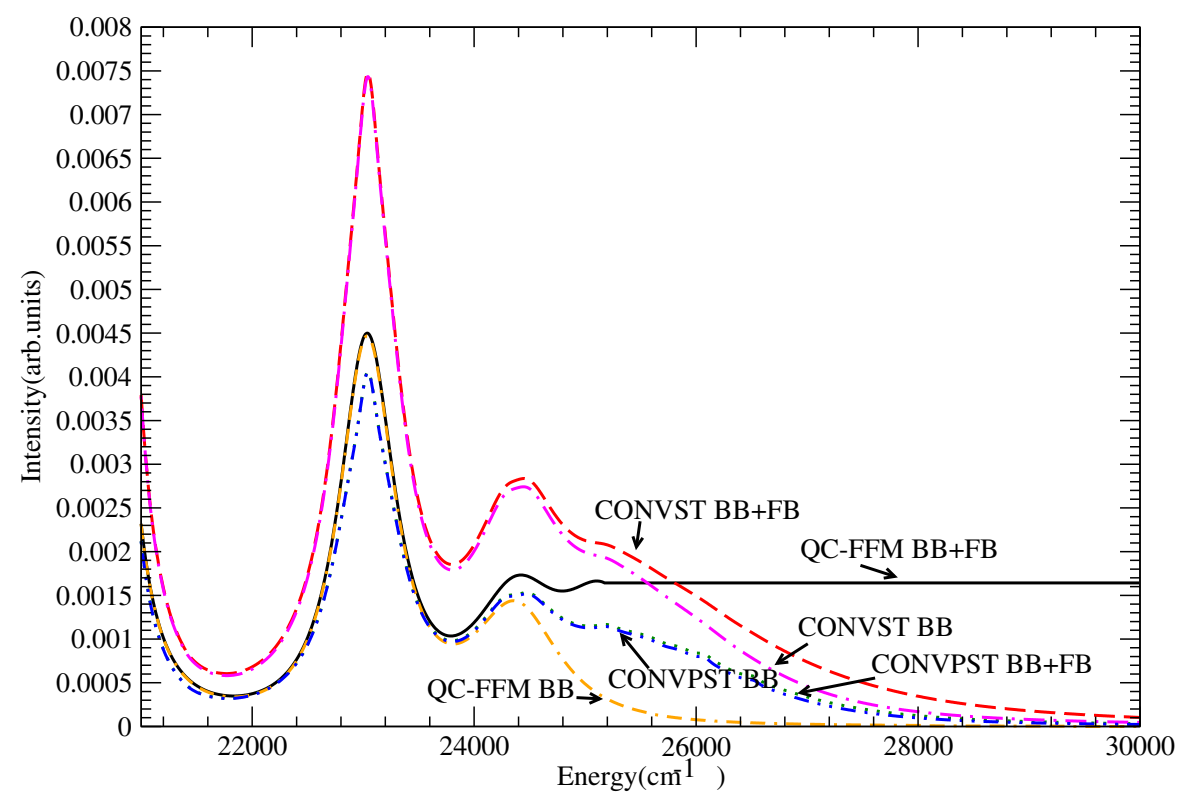

Figure 25. BB + FB vs. BB comparison for the lowest density close to the continuum. Shown are QC-FFM for BB + FB (solid) and BB only (dot-double dashed), CONVST for BB + FB (dashed) and CONVST for BB only (dash-dotted) and CONVPST for FB + BB (dotted) and BB only (dash-double dotted).

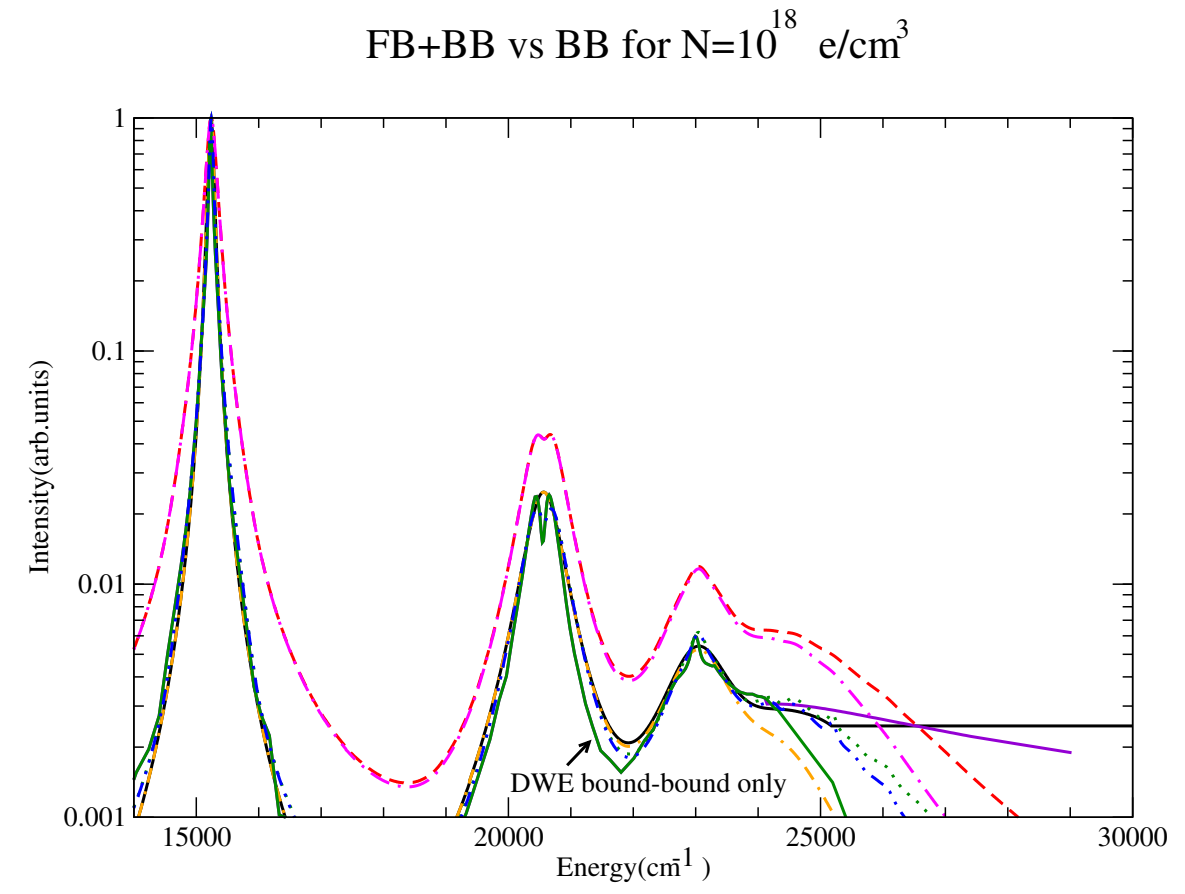

Figure 26. $\mathrm{BB}+\mathrm{FB}$ vs. $\mathrm{BB}$ comparison for the intermediate density close to the continuum. Shown are QC-FFM for BB + FB (solid) and BB only (dot-double dashed), CONVST for BB + FB (dashed) and CONVST for BB only (dash-dotted) and CONVPST for FB + BB (dotted) and BB only (dash-double dotted). Also shown is DWE (green solid) for the BB spectrum. 
Figure 27 shows the region close to the continuum for the highest density run. Again, QC-FFM displays a flat line, which is not seen for the other codes.

$$
\mathrm{FB}+\mathrm{BB} \text { vs } \mathrm{BB} \text { for } \mathrm{N}=3 \times 10^{18} \mathrm{e} / \mathrm{cm}^{3}
$$

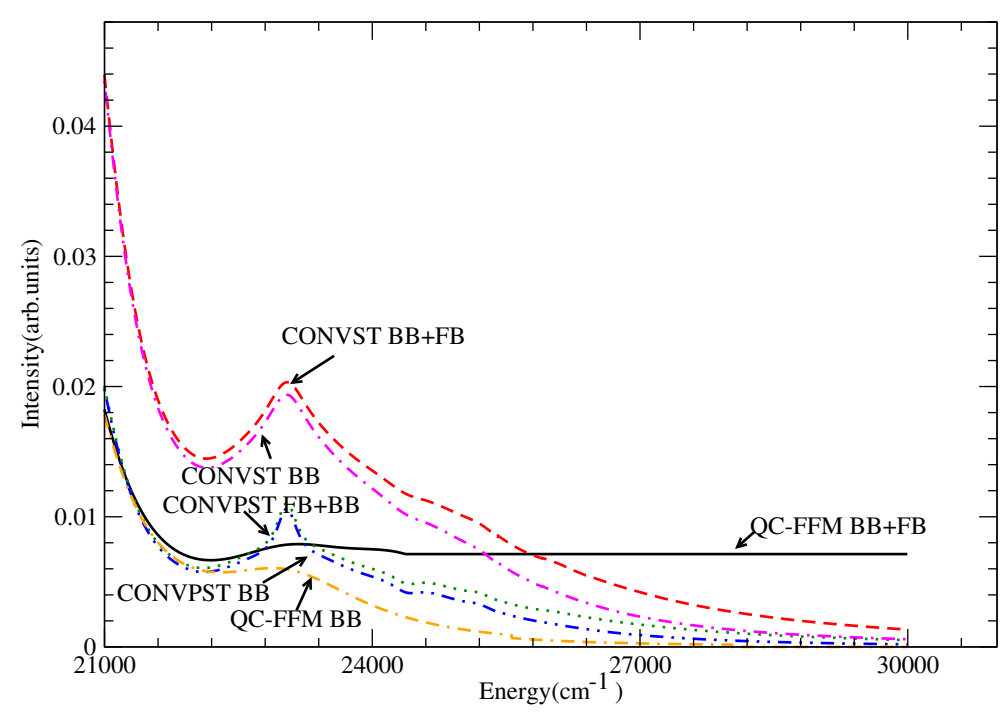

Figure 27. $\mathrm{BB}+\mathrm{FB}$ vs. $\mathrm{BB}$ comparison for the highest density close to the continuum. Shown are QC-FFM for BB + FB (solid) and BB only (dot-double dashed), CONVST for BB + FB (dashed) and CONST for BB only (dash-dotted) and CONVPST for FB + BB (dotted) and BB only (dash-double dotted).

The constant baseline is not seen in CONVST/PST and this is understandable: Figure 28 displays CONVST individual lines for $N=3 \times 10^{18} \mathrm{e} / \mathrm{cm}^{3}$ and $n=8-17$. It is seen than while the higher $n$ lines are indeed flat, they have much smaller intensity than the lower lines, even at the high energy wings. Therefore, they will not significantly affect the line profile, even close to the series limit. The same effect is seen for PST in Figure 29.

Hydrogen Balmer Series intensity comparison for CONVST

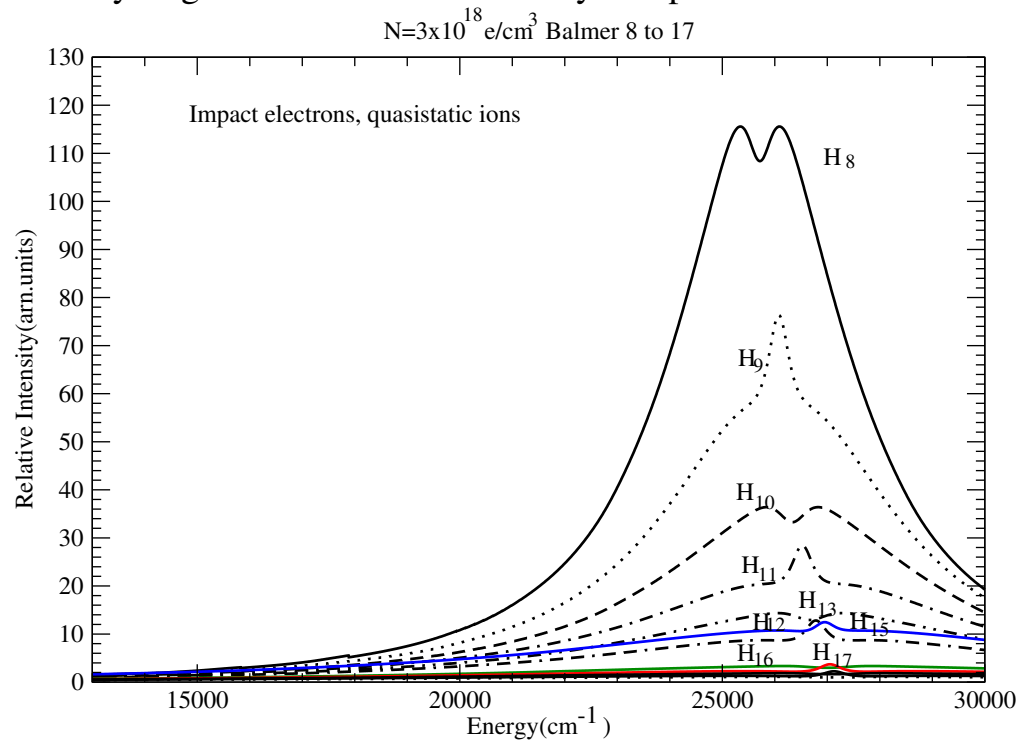

Figure 28. Intensity comparison for Balmer 8 to Balmer 17 at the highest density. Shown are CONVST profiles for $H_{8}$ (solid black), $H_{9}$ (dotted), $H_{10}$ (dashed), $H_{11}$ (dash-dotted), $H_{12}$ (dash-double dotted), $H_{13}$ (dot double dashed), $H_{15}$ (solid blue), $H_{16}$ (solid green) and $H_{17}$ (solid red). 


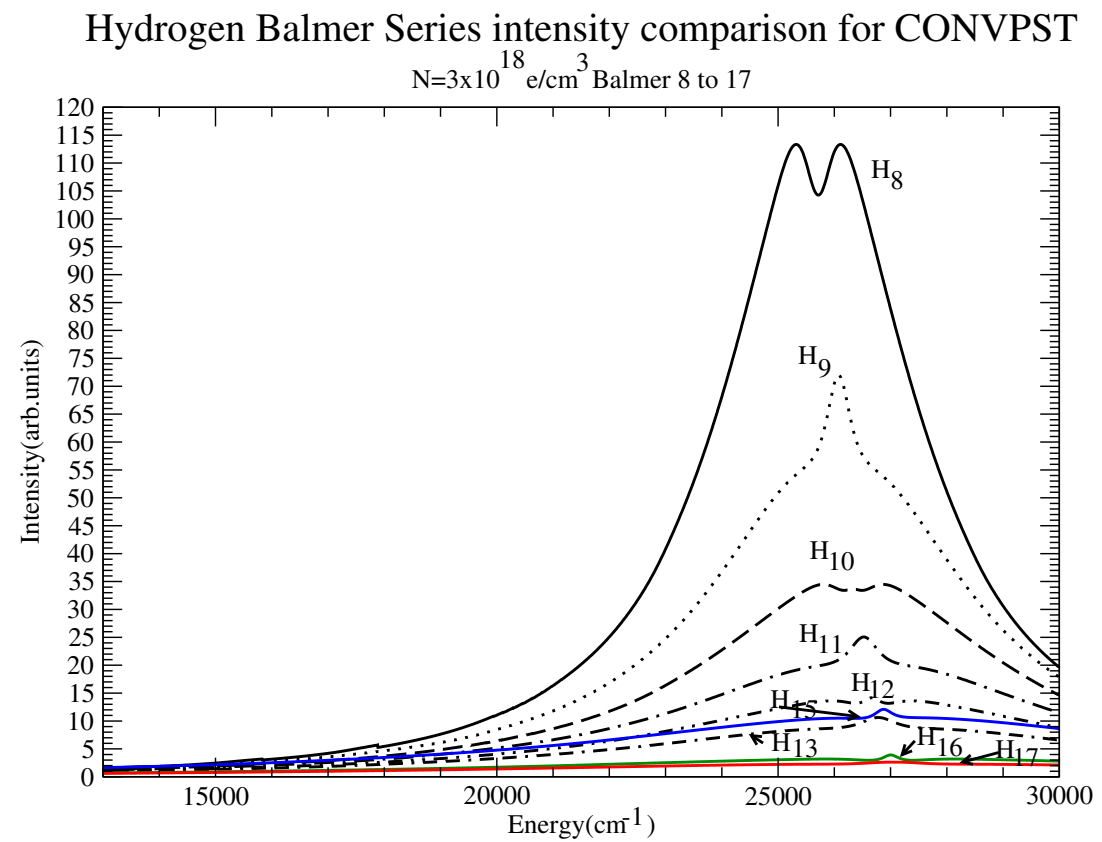

Figure 29. Intensity comparison for Balmer 8 to Balmer 17 at the highest density. Shown are CONVPST profiles for $H_{8}$ (solid black), $H_{9}$ (dotted), $H_{10}$ (dashed), $H_{11}$ (dash-dotted), $H_{12}$ (dash-double dotted), $H_{13}$ (dot double dashed), $H_{15}$ (solid blue), $H_{16}$ (solid green) and $H_{17}$ (solid red).

These results are in doubt, however, because the effects of incomplete collisions on the line wings have not been accounted for. Figure 30 is essentially Figure 26 with the CONV runs now using the Lewis cutoff. However, these runs that include the Lewis cutoff also do not show a flat baseline for CONV.

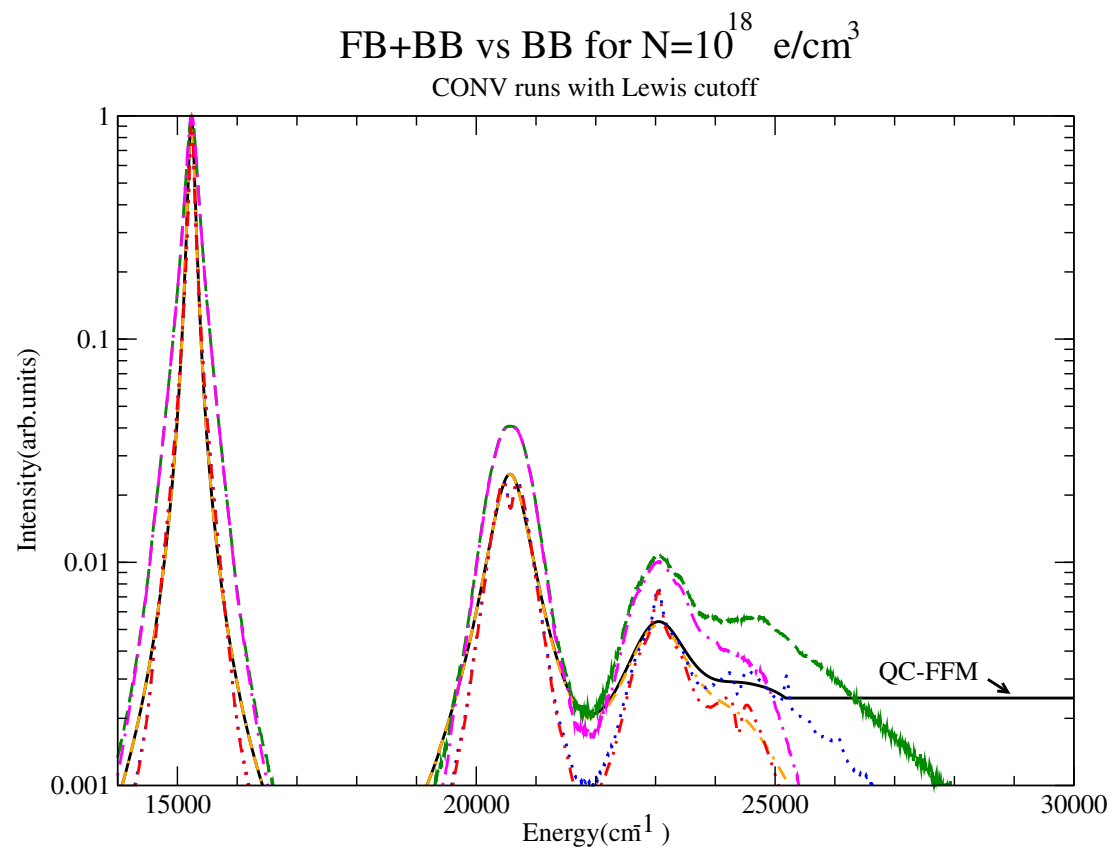

Figure 30. $\mathrm{BB}+\mathrm{FB}$ vs. $\mathrm{BB}$ comparison for the intermediate density close to the continuum. Shown are QC-FFM for BB + FB (solid black) and BB only (dot-double dashed orange), CONVST for BB + FB (dashed green) and CONVST for BB only (dash-dotted magenta) and CONVPST for FB + BB (dotted blue) and BB only (dash-double dotted red). The CONV runs include strong collisions and the Lewis cutoff. The Inglis-Teller cutoff was used in the BB only CONV results. 
The reason for CONV not obtaining a flat baseline is the same as explained above: Namely for both ST and PST, the wings in CONV are still dominated by the lower lying lines. This is illustrated in Figures 31 and 32 for CONVST and CONVPST respectively, both with the Lewis cutoff and strong collision term. It is seen that although the higher series members exhibit very flat wings, these wings are still dominated by the wings of the lower-lying lines. In both cases, the noise in the spectra arises from the extraction procedure, i.e., to obtain the $H_{n}$ profile, the total profile of the entire series up to the $(n-1)$ th upper level was subtracted from the the total profile of the entire series up to the nth upper level.

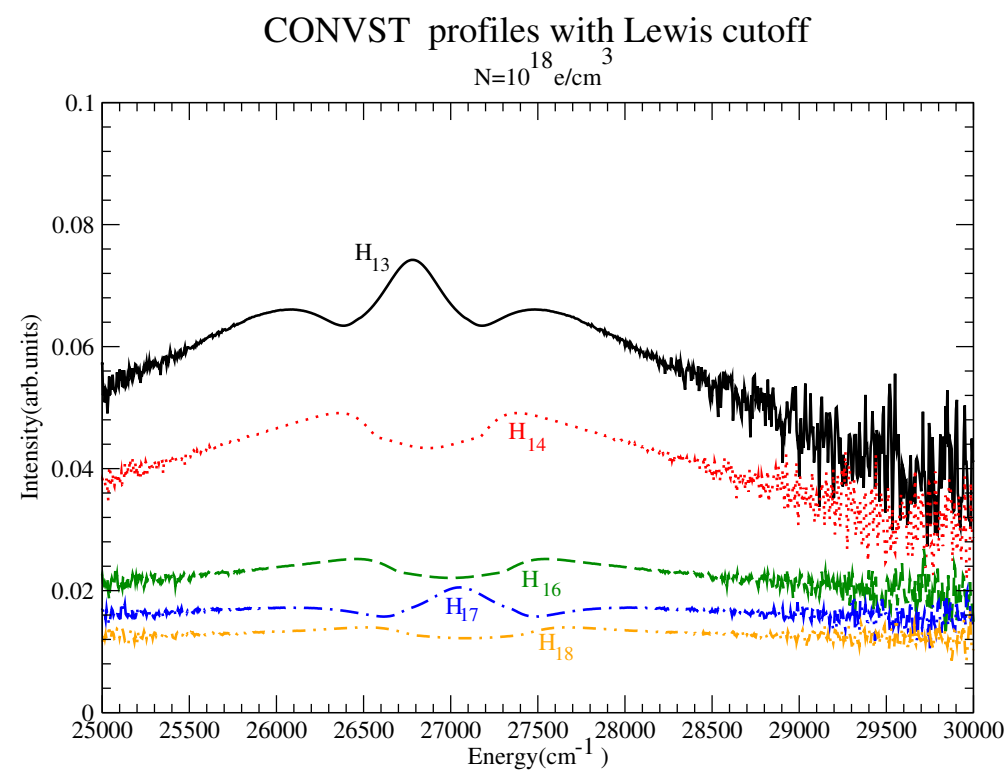

Figure 31. Intensity comparison for Balmer 13 to Balmer 18 at the intermediate density. Shown are CONVST profiles for $H_{13}$ (solid black), $H_{14}$ (dotted red), $H_{16}$ (dashed green), $H_{17}$ (dash-dotted blue) and $H_{18}$ (dash-double dotted orange).

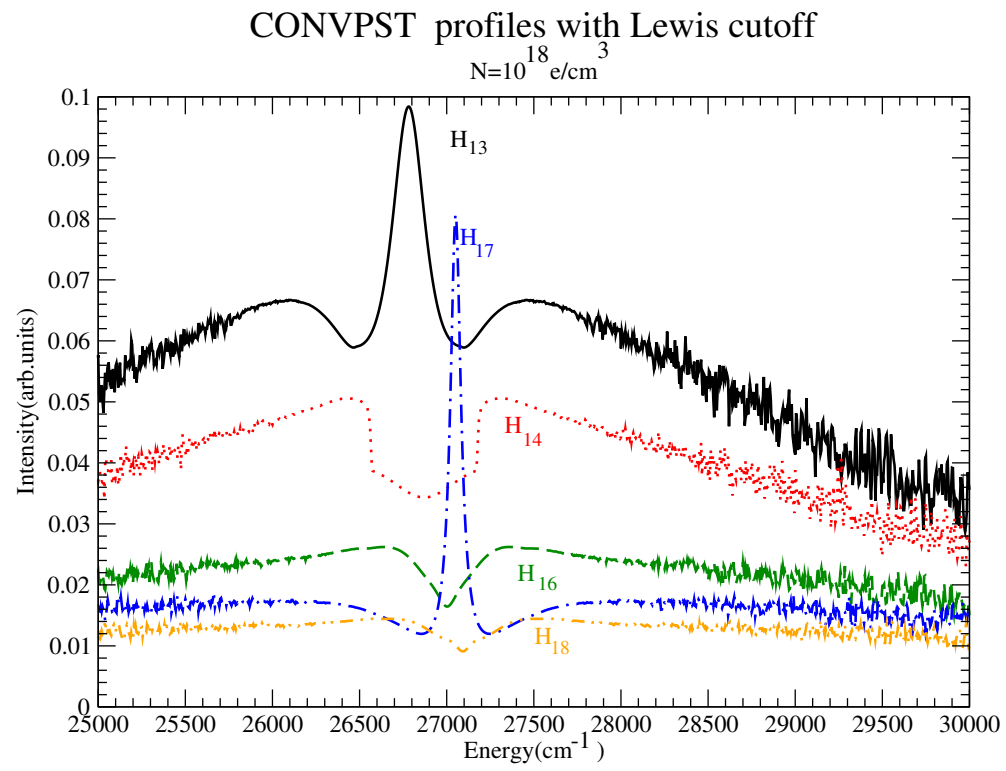

Figure 32. Intensity comparison for Balmer 13 to Balmer 18 at the intermediate density. Shown are CONVPST profiles for $H_{13}$ (solid black), $H_{14}$ (dotted red), $H_{16}$ (dashed green), $H_{17}$ (dash-dotted blue) and $H_{18}$ (dash-double dotted orange). 


\section{Discussion}

With respect to the bound-bound transitions, the present comparison confirms the importance of strong collisions, even for $H_{\alpha}$ at the lowest density as well as penetrating collisions, which significantly reduce the strong collision phase space [9]. This is evidenced by the good agreement of DWE and CONVPST which both account for penetrating collisions. Interestingly enough, QC-FFM, which does not account for penetration or strong collisions, is in good agreement with the above two codes, which do. The present work provides no definitive answer to why this is so, possibly because of the limited number of comparisons made for this case. Especially with regard to the higher series members, differences in width computations can be very important for the code decision to include or exclude a level. Since these lines have widths that are often larger than the plasma frequency, inclusion of incomplete collisions is important. With regard to whether the addition of levels beyond the series upper level computed by the codes (e.g., via Inglis-Teller estimates), it is shown that this can be a flat line, provided the contribution of lower lying transitions is negligible at such $\Delta \omega$. If the wings of the lower-lying transitions are appreciable, or even dominant, due to their higher intensity, this exactly flat line is not realized.

\section{Conclusions}

For the computation of entire series, important physics includes the following:

- $\quad$ Strong and penetrating collisions.

- $\quad$ The method for determining the maximum upper level $n^{*}$.

- In impact calculations, correctly accounting for incomplete collisions (e.g., via the unified theory) is important, especially for high $n$-lines.

These aspects were described and their effect tested in detail. In particular, the importance of ion dynamics (not important here), strong and penetrating collisions (clearly relevant here) and incomplete collisions (also clearly relevant) are identified. The codes differ in their handling of these important aspects. Specifically there are two codes that do not explicitly treat penetrating collisions, but treat (via the FFM) ion dynamics. One of them (QC-FFM) has no notion of strong and weak collisions, while the other (PPP) treats strong collisions via a strong collision term or estimate, as does CONV. DWE is a new quantum code that includes penetrating collisions and does not use a strong collision term, but on the other hand it is a perturbative treatment, as is CONV. PPP also uses a strong collision estimate, while QC-FFM relies on the FFM for dynamic aspects, which cannot handle the impact regime very well. With respect to $n^{*}$, PPP and DWE make no attempt to estimate it from theory and use instead a "large enough" $n *$. QC-FFM uses the Inglis-Teller limit, while CONV has different options, with the Inglis-Teller one of them, although another model was mostly used as the default to check the effect. The choice of $n^{*}$ was found to be important towards the series limit. Finally, with respect to incomplete collisions, this is built-in in DWE and QC-FFM (via FFM), it is also in principle available in PPP, but this option was not used for the workshop runs. CONV also has this as an option and runs were done with and without the Lewis cutoff. This was predicted and found to also be important.

The workshop's aim is to identify areas of code agreement as well as areas and reasons for disagreements. All codes involve approximations and/or choices and tradeoffs and, except for ion dynamics, these approximations or choices were shown to be important in the final profile. The codes involve different run-times, which makes fast codes like QC-FFM and PPP more appropriate for large-scale calculations. As a result, the authors do not claim that any code is "better" or more appropriate in general.

Acknowledgments: The support of IAEA is gratefully aknowledged. The work of E.S. was partly supported by the Israel Science Foundation. Sandia National Laboratories is a multimission laboratory managed and operated by National Technology \& Engineering Solutions of Sandia, LLC, a wholly owned subsidiary of Honeywell International Inc., for the U.S. Department of Energy's National Nuclear Security Administration under contract DE-NA0003525. The views expressed in the article do not necessarily represent the views of the U.S. Department of Energy or the United States Government. 
Author Contributions: All authors contributed equally to the work reported.

Conflicts of Interest: The authors declare no conflict of interest.

\author{
Abbreviations \\ The following abbreviations are used in this manuscript: \\ BB Bound-Bound Transitions \\ FB Free-Bound Transitions
}

\title{
References
}

1. Inglis, D.R.; Teller, E. Ionic Depression of Series Limits in One-Electron Spectra. Astrophys. J. 1939, 90, 439.

2. Alexiou, S. Enhancement of line broadening in plasmas by penetrating collisions for hydrogenlike lines. High Energy Density Phys. 2017, 23, 188-194.

3. Salzmann, D.; Stein, J.; Goldberg, I.B.; Pratt, R.H. Effect of nearest-neighbor ions on excited ionic states, emission spectra, and line profiles in hot and dense plasmas. Phys. Rev. A 1991, 44, 1270-1280.

4. Alexiou, S. Scaling of hydrogen electron stark widths at high densities and the Inglis-Teller limit. High Energy Density Phys. 2009, 5, 68-73.

5. Calisti, A.; Mossé, C.; Ferri, S.; Talin, B.; Rosmej, F.; Bureyeva, L.A.; Lisitsa, V.S. Dynamic Stark broadening as the Dicke narrowing effect. Phys. Rev. E 2010, 81, 016406.

6. Bureeva, L.A.; Kadomtsev, M.B.; Levashova, M.G.; Lisitsa, V.S.; Calisti, A.; Talin, B.; Rosmej, F. Equivalence of the method of the kinetic equation and the fluctuating-frequency method in the theory of the broadening of spectral lines. JETP Lett. 2010, 90, 647-650.

7. Stambulchik, E.; Maron, Y. Stark effect of high-n hydrogen-like transitions: Quasi-contiguous approximation. J. Phys. B 2008, 41, 095703.

8. Stambulchik, E.; Maron, Y. Quasicontiguous frequency-fluctuation model for calculation of hydrogen and hydrogenlike Stark-broadened line shapes in plasmas. Phys. Rev. E 2013, 87, 053108.

9. Alexiou, S.; Poquérusse, A. Standard line broadening impact theory for hydrogen including penetrating collisions. Phys. Rev. E 2005, 72, 046404.

10. Poquérusse, A.; Alexiou, S. Penetrating standard line broadening impact theory for hydrogen-like ions. J. Quant. Spectrosc. Radiat. Transf. 2006, 99, 493-498.

11. Gomez, T.A. Improving Calculations of the Interaction between Atoms and Plasma Particles and Its Effect on Spectral Line Shapes. Ph.D. Thesis, University of Texas at Austin, Austin, TX, USA, 2017.

12. Gomez, T.A.; Nagayama, T.; Kilcrease, D.P.; Fontes, C.J.; Montgomery, M.H.; Winget, D.E. Penetrating Collisions by Electrons and its Effect on Electron Broadening. 2018, in preparation.

13. Woltz, L.A.; Hooper, C.F. Full Coulomb calculation of Stark broadening in laser-produced plasmas. Phys. Rev. A 1984, 30, 468.

14. Griem, H.R.; Blaha, M.; Kepple, P.C. Stark-profile calculations for Lyman-series lines of one-electron ions in dense plasmas. Phys. Rev. A 1979, 19, 2421.

15. Alexiou, S.; Lee, R.W. Semiclassical calculations of line broadening in plasmas: Comparison with quantal results. J. Quant. Spectrosc. Radiat. Transf. 2006, 99, 10-20.

16. Lewis, M. Stark Broadening of Spectral Lines by High-Velocity Charged Particles. Phys. Rev. 1961, $121,501$.

17. Ferri, S.; Calisti, A.; Stamm, R.; Talin, B.; Lee, R.W.; Klein, L. Electronic broadening model for high-n Balmer line profiles. Phys. Rev. E 1998, 58, R6943.

(C) 2018 by the authors. Licensee MDPI, Basel, Switzerland. This article is an open access article distributed under the terms and conditions of the Creative Commons Attribution (CC BY) license (http:/ / creativecommons.org/licenses/by/4.0/). 